Journal of Al Azhar University Engineering Sector

Vol. 11, No. 38, January, 2016, 59-83

\title{
EXPERIMENTAL INVESTIGATION OF THE PERFORMANCE OF FIVE LAYERED GAS TO GAS HEAT EXCHANGER WITH A POROUS MEDIUM
}

\author{
H. Z. Barakat ${ }^{1}$, N. A. Mohammed ${ }^{2}$, and N. Anis ${ }^{3}$ \\ Mechanical Power Dept., Faculty of Engineering, Ain Shams University, Cairo, Egypt
}

\begin{abstract}
This paper presents the performance of a simple five-layers-type gas to gas heat exchanger, which is simple to construct, easy to maintain and has low space requirements, under different laminar gaseous flow rates and temperatures. The merits and drawbacks of the use of porous material inserts to improve the performance of this type of heat exchanger are investigated. An experimental work was carried out to investigate the influence of the insertion of porous segments with different thicknesses on heat transfer enhancement and the associated pressure drop as these are the many determining factors in most engineering applications. During these experiments the volume flow rates of the hot and cold air streams were varied such that the volume flow rate of both of the streams was the same in any of the experimental runs. These experiments were conducted for different values of hot air inlet temperature, while keeping the cold air inlet temperature nearly constant. Also the results obtained in this work was compared with the corresponding results obtained previously using a similar three layered heat exchanger under similar operating hydro-thermal conditions. The investigations showed that the thermal performance of the heat exchanger due to the two additional layers was pronouncedly improved on the expense of an increase in pressure drop of much lower cost.
\end{abstract}

\section{Introduction}

The increase in energy cost and energy consumption has required more effective use of energy. The problem of dissipating high heat rates has received much attention and efforts are continuously made to restore the energy exhausted from many industrial applications through using different types of heat exchangers. Considerable researches have been devoted to increase heat transfer rates in heat exchangers by implementing passive enhancement methods that require no direct consumption of external power. On the basis of both theoretical and experimental analysis, it was concluded that effective heat transfer enhancement can be reached by the use of porous structures like metallic foam in heat exchangers especially if the gas is the working fluid, which are inexpensive techniques to extend the heat transfer area, flow redistribution, heat capacity of the fluid-porous material medium and provide more effective modes of energy exchange. As it is the case in the high temperature applications, the conversion of the flowing gas enthalpy absorbed by the porous media which is emitted by it as a radiant thermal energy that is absorbed by the enclosure of recuperative type heat exchangers, makes it more effective technique of heat recovery. However, using porous materials increases the pressure drop across the heat exchanger and thus increases the power required to drive the fluids.

The Use of porous media to increase the heat transfer was studied numerically. Also Lots of experiments have been .carried to study the effect of inserting porous media on heat transfer enhancement. Mehmet SÖzen et al [2] described modeling and numerical simulation of fluid flow and 
heat transfer in tubes filled with rolled copper mesh- as a porous inserts - brazed to the tube walls using water as the energy transport fluid. They found that enhancement in heat transfer was at the cost of a large pressure drop of such magnitude that may not be allowable in certain applications. A. A. Mohamad [3] investigated numerically the heat transfer enhancement for air flow in a pipe or a channel fully or partially filled with porous medium with a constant wall temperature due to the flow redistribution, thermal conductivity modification and radiative property modification of the medium. Their results showed that partially filling the conduit with porous medium has two advantages: it enhances the rate of heat transfer, and the pressure drop is much less than that for a conduit fully filled with a porous medium. Horng et al [4] solved numerically the unsteady flow and convection heat transfer of laminar forced fluid flow across the porous square cylinder with the heated cylinder bottom in the middle of the channel, adopting the general Darcy-Brinkman Forchheimer model for the porous region. The results indicate that the average local Nusselt number increases as the Reynolds number increases; in particular, the increase is more obvious at a higher Darcy number while the porosity has slight influence on heat transfer. Arunn Narasimhan et al [5] analyzed numerically the flow and heat transfer in parallel plate channels containing distributed heated solid blocks mimicking electronics and cooled by forced convection, using direct approach and using the Bi-Disperse Porous Medium approach which provided a viable method of thermo-hydraulic performance enhancement. $H$. Shokouhm et al [6] studied the effect of porous insert position whether attached to the channel walls or in the channel core on enhanced heat transfer in a parallel-plate channel partially filled with a fluidsaturated porous medium. It was found out that with a porous layer located in the channel core, pressure loss was higher than that of the case with porous medium adjacent to the walls. When the thermal conductivity and Darcy number of porous media are high, locating the inserts near the walls is superior and vice versa for lower Darcy Numbers. It is also remarkable that the required pumping power increases at lower Darcy numbers. Y. Tian et al [7] investigated the effects of metal foams on heat transfer enhancement in phase change materials using two-equation non-equilibrium heat transfer model. Numerical results showed that the addition of metal foams considerably enhanced the heat transfer performance (about 10 times) and also indicated that metal foams with smaller pore size and porosity has better heat transfer performance than those with larger pore size and porosity. The numerical results are validated by experimental data. Shiang-Wuu Perng et al [8] studied numerically how various parameters affect the heat transfer enhancement and vortex-induced vibration by installing a porous vortex-generator in a block-heated channel. The results indicate that heat transfer enhancement and vortex-induced vibration increase with increasing Reynolds number and width-toheight ratio. However, the porosity has slight influence on heat transfer enhancement and vortexinduced vibration. Saman Rashidi et al [9] investigated numerically the flow-field and heat transfer around a cylinder embedded in a layer of homogeneous porous media. Volume averaged equations are used for modeling transport phenomena within the porous layer and conservation laws of mass, momentum and energy are applied in clear region. The numerical results indicate that the presence of a porous layer around the cylinder increases the wake length with decreasing the Darcy number while the critical radius of insulation increases. Therefore, for heat transfer enhancement techniques, it is more suitable to choose permeable porous layer with high thermal conductivity. A. Abou el Mehrizi et al [10] used the lattice Boltzmann method to investigate the heat transfer enhancement in a ventilated porous media plate heat exchanger. The heat exchanger is modeled by a square cavity with inlet and outlet thermally insulated ports and three hot fins with constant temperature. The BrinkmanForchheimer model was used to simulate the porous domain. The results showed that reducing the porosity increases the mean temperature of outlet flow, so the thermal efficiency of the heat exchanger increases and determined the best location for maximum outlet temperature. Yasser Mahmoudi et al [11] examined numerically the heat transfer enhancement in a pipe partially filled with a porous medium. Darcy-Brinkman-Forchheimer model was utilized to model the flow inside the porous material. Energy equations for both solid phase and fluid phase in the porous medium were solved through local thermal non-equilibrium model. The effects of inertia term on the Nusselt number and pressure drop are further studied. For a given model and for Darcy number $<10^{-3}$, the Nusselt number is found independent of Forchheimer number but for Darcy number $>10^{-3}$ as Forchheimer number increases the computed Nusselt number increases. Entropy generation and convective heat transfer 
through a pipe partially filled with a porous material was numerically studied by Mahboobe Mahdavi et al [12] using finite volume SIMPLER algorithm and Darcy-Brinkman-Forchheimer model in the porous region if the cylindrical porous material is placed at the core or if it is attached to the inner wall. The results proved that the position of the porous medium has considerable effect on the thermal performance of the pipe and that the thermal conductivity ratio has significant effect on increasing enhanced heat transfer in first case. Numerical simulations have been carried out by FumeiRong et al [13] to investigate the heat transfer enhancement for fluid flow in a pipe partially filled with porous media. The new axi- symmetric lattice Boltzmann model is used to calculate the fluid flow and heat transfer characteristics. The results showed that inserting porous medium can effectively improve heat transfer; however increasing the flow resistance.

The concept of micro-channel heat sinks was introduced by Pei-Xue Jiang et al [14] by investigating experimentally fluid flow and forced convection heat transfer in micro-heat-exchangers with either micro-channels or porous media. The deep micro-channel design offers a better overall performance than either the porous media or shallow micro-channel alternatives. Pei-Xue et al [15] investigated experimentally forced convection heat transfer of water and air in sintered porous plate channels. The effective thermal conductivity of the sintered porous media was found to be much higher than for nonsintered porous media due to the improved thermal contact caused by the sintering process. Kang-Hoon Ko et al [16] carried out an experimental investigation to measure module average heat transfer coefficients in uniformly heated rectangular channel with wall mounted porous baffles. The use of porous baffles resulted in heat transfer enhancement as high as 300\% compared to heat transfer in straight channel with no baffles. However, the heat transfer enhancement per unit increase in pumping power was less than one for the range of parameters studied in this work. Correlation equations were developed for heat transfer enhancement ratio and heat transfer enhancement per unit increase in pumping power in terms of Reynolds number. N. Delalic [17] described the usage of boiler`s burner with built-in heat exchanger as a method of development of heat exchangers -using air as the working fluid- on the basis of porous medium whose specific thermal load reaches about $7 \mathrm{MW} / \mathrm{m}^{3}$. C. Hutter, D.B et al [18] studied experimentally the influence of the geometry of the structure porous inserts on the heat transfer performance for commercially available metal foam for air and water. An increase of the convective heat transfer with increasing pore size, ascribed to the bigger ligament thickness, could be observed. Also the shape of the ligaments was found to have a huge influence on the heat transfer performance. The effect of a fixed wall connection was studied by the aid of reference geometry with and without wall connection. A big difference in the heat transfer rate was found. In case of the fully sintered device the pseudo-convective heat transfer rate was found to be in average $30 \%$ higher compared to the sintered structure without wall connection. T. Tomimura [19] had proposed a multilayered type of gas to gas heat exchanger (2,3 and 5 Layers) using porous media and studied the effective energy conversion method between flowing gas enthalpy and thermal radiation. Bogdan I. Pavel et al [20] investigated experimentally and numerically the effect of metallic porous materials, inserted in a pipe, on the rate of heat transfer. The results obtained lead to the conclusion that higher heat transfer rates can be achieved using porous inserts at the expense of a reasonable pressure drop. Hetsroni et al [21] investigated experimentally the heat transfer and pressure drop in a rectangular channel with sintered porous inserts made of stainless steel of different porosity. Estimating the efficiency of sintered porous heat sink and the comparison with aluminum compressed foams, show that such heat sink gives very high value of the heat transfer performance but required a drastic increase in the pumping power. S. A. Gandjalikhan Nassab [22] provided a five layered heat exchanger equipped with porous material. One of the layers was introduced as porous radiant burner which is one of the other applications of porous materials in thermal systems. Numerical results showed a very high efficiency for this type of porous air heater. Z.F. Huang [23] developed a porous media with a slightly smaller diameter to a tube and inserted it in the core of the tube under the constant and uniform heat flux condition. The experimental and numerical results showed that the heat transfer is enhanced greatly with an acceptable flow resistance increase proving that the core flow enhancement is an effective method for enhancing heat transfer. G. Venugopal [24] examined the potential of a simple and inexpensive porous insert consisting of a stack of metallic perforated plates filled inside a vertical rectangular air duct under forced flow conditions. The experimentally measured non-dimensional 
parameters are correlated for predicting the heat transfer performance. Chainarong Chaktranond et al [25] experimentally investigated the influences of electrical voltage, particle sizes and layered arrangement on the heat and mass transfer in porous packed bed subjected to electro-hydrodynamic drying. The results show that the convective heat transfer coefficient and drying rate are enhanced considerably with a Corona wind. Khaled Al-Salem et al [26] carried out an experimental study to investigate the effects of porosity and thickness of porous sheets on heat transfer enhancement in a cross flow over heated cylinder; by wrapping an aluminum porous sheet over a circular tube in a heat convection configuration. It was observed that heat transfer is greatly enhanced with the addition of the porous layers. Also, the addition of the porous layers didn't appear to increase the pressure loss.

\section{The Scope of This Work}

This paper presents the results of the experimental investigation of the thermo-hydraulic performance of a five layered gas to gas heat exchanger when it was separately run without and with a porous segment inserted in each layer. The thermo-hydraulic performance includes essentially the exit temperatures of both of the cold and hot gas streams, the heat recovery rate the heat recovery ratio, the heat losses percentages and the cold air side pressure drop through the heat exchanger at different hot and cold air volume flow rates, hot air inlet temperatures for the heat exchanger without and with the porous medium of various thicknesses. The result obtained for the runs without the porous segments, which are necessary to indicate the effect of using the porous segments are carried out under the same hydro-thermal conditions and are plotted on the relevant figures on the abscess as zero porous segment. These results clearly show the effect of the installation of the porous medium on enhancing the heat transferred, increasing the heat recovery ratio and the corresponding rise in the pressure drop. The results obtained during these investigations are compared with those obtained previously using a three layered heat exchanger under the same operating conditions to examine the effect of increasing the number of layers on the hydrodynamic and thermal parameters of this type of heat exchanger under the different operating conditions.

\section{THE EXPERIMENTAL APPARATUS}

\subsection{The Test Section}

The present experimental work is carried out on a heat exchanger which consists of five chambers stacked one atop each other forming the five layered gas to gas type heat exchanger through which heat is exchanged between two streams of cold and hot gases. The cold gas stream passes through three chambers which are usually referred as the heat recovery sections. It enters the heat exchanger into the top chamber which is the first heat recovery section HR1. It leaves this heat recovery section to the third chamber which is the second heat recovery section HR2. The cold air leaves HR2 section to the third heat recovery section HR3 that is the lowest chamber from which it flows out at its highest temperature as shown in figures 1 and 2.

The hot air stream flows into the other two chambers which are denoted the high temperature sections HT1 and HT2. It enters the first high temperature section HT1 which is the second chamber at a temperature $T_{h, i}$ and leaves it to the second high temperature section HT2 which is the fourth chamber at exit temperature $\mathrm{T}_{\mathrm{h}, \mathrm{o}}$. Figure 1 shows the layout of these chambers atop each other and demonstrates the relative position of the cold air heat recovery sections with respect to the hot air high temperature sections. The figure also shows the elbows and piping connecting the cold air chambers and also those connecting the hot air chambers.

Briefly, the heat exchange between the hot and the cold air streams stakes place through the four horizontal metallic walls of the chambers which separate the two air streams and no merging of them takes place. In this case, heat exchange takes place by convection between the gases and the separating walls as well as by radiant heat exchange between the porous inserts and the walls of the hot air chambers and from both of them by radiation also to the separating walls. The convective and radiative heat fluxes are transferred through the thin separating walls by conduction and from it by convection to the cold air in contact with the other side of the separating walls.

The three layered heat exchanger whose results are compared to the present results consists of a two cold air heat recovery chambers sandwiched between them vertically one hot air chamber. The 
construction and the size of these chambers and the porous segments are the same in both of these heat exchangers.

Each chamber is constructed from steel sheets of $4 \mathrm{~mm}$ thickness. The cross section area of each chamber is $200 \mathrm{~mm} \times 200 \mathrm{~mm}$. Each chamber has an inlet and exit ducts of a $(50 \mathrm{~mm} \times 200 \mathrm{~mm})$ cross section area. The layered chambers are separated from each other by $270 \mathrm{~mm} \times 270 \mathrm{~mm}$ steel plates of $4 \mathrm{~mm}$ thickness. Heat-resisting black paint is applied on the inside surface of each chamber to improve the radiation characteristics of its internal surfaces, mainly increasing both of the absorptivity and the emissivity of these surfaces. The external sides of the chambers, the connecting ducting and elbows are insulated by rock wool blankets of $70 \mathrm{~mm}$ thickness.

In case of inserting porous segments, each chamber is provided by a segment of the same porous material, and of the same size as those of the others, which is represented by the rectangle shown by pixel pattern in Fig. 3. The porous material plate placed in each section is made of foamed alumina alloy. The porosity and specific surface areas of the porous plate are $88 \%$, and $1700 \mathrm{~m}^{2} / \mathrm{m}^{3}$, respectively. The porous segment is placed in the middle of the height of each chamber. The clearances between the porous plate and each of the top and bottom surfaces of the chamber are equal; to serve as inlet and outlet passages of the gas stream inside each chamber as depicted in figure 3.

\subsection{Essential Equipment}

\subsubsection{Air Supply System}

An air supply system is provided that uses a centrifugal air blower which has a circular cross section exit of a diameter of (two inches, 2"), rotating at $2900 \mathrm{rpm}$ to produce a maximum air pressure of 0.07 bar and maximum volume flow rate of $128 \mathrm{~m}^{3} / \mathrm{hr}$. The air blower is driven by a single phase electric motor $(220 \mathrm{~V}, 1.5 \mathrm{Hp}, 2900 \mathrm{rpm})$.

\subsubsection{Electric Heater}

The hot air, which is supplied to the first high temperature chamber HT1 by the same blower through a regulating valve, passes through a circular duct to the electric heater. The heater has four electric heating elements, each one is of $1.8 \mathrm{~kW}$ capacity at $220 \mathrm{~V}$. The electric heater output is varied using two variable transformers of a maximum power of $8 \mathrm{~kW}$ each in order to adjust the temperature of the hot air entering the heat exchanger at the desired temperature from $100{ }^{\circ} \mathrm{C}$ to $500{ }^{\circ} \mathrm{C}$. The heated air is passed into the second chamber which is the first high temperature section HT1 to flow through its porous segment and is discharged through the connecting piping to the fourth chamber which is the second high temperature section HT2 and from there it flows out into the atmosphere as shown in figures (1) and (3). 


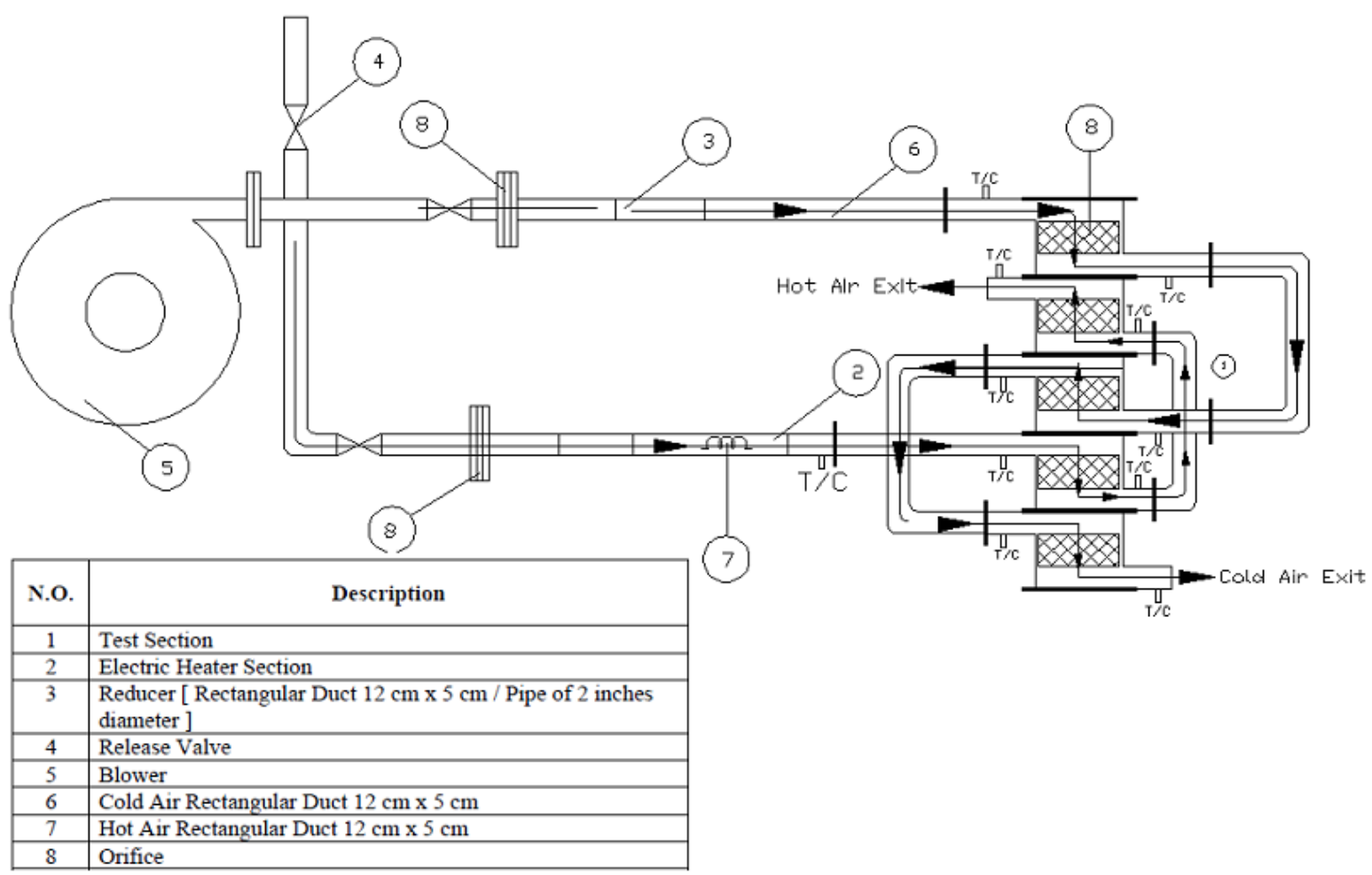

Fig. (1) Schematic diagram of experimental apparatus of multi-layered radiant type gas to gas heat exchanger

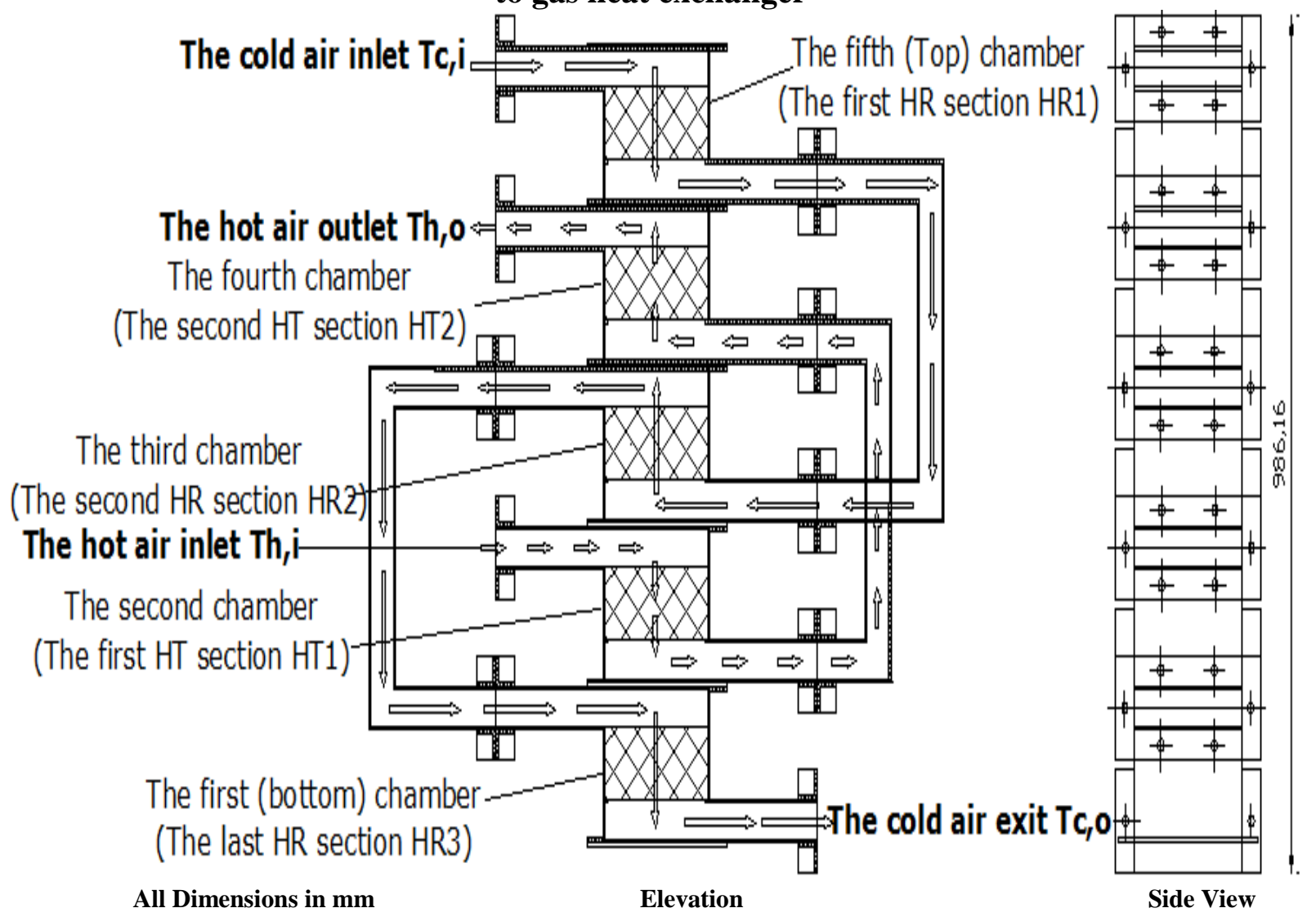


Fig. (2) Schematic Layout of The Five layers Test Section assembly
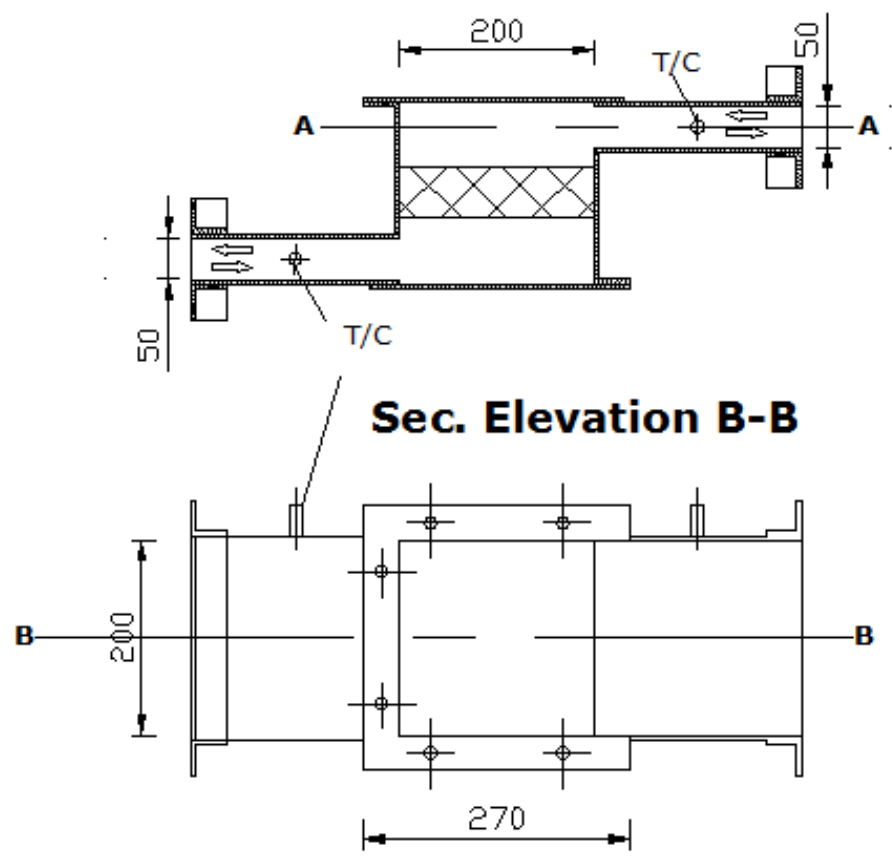

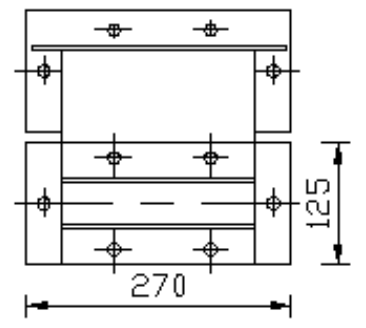

Side View

Sec. Elevation A-A

All Dimensions in mm

Fig. (3) Schematic View of an Intermediate Chamber

\subsubsection{Measurement Instruments}

The air streams temperatures along the axes perpendicular to the air flow direction are measured to calculate the heat recovery rate and other thermal performance of the heat exchanger. The air temperatures are measured using a thermocouples type $\mathrm{K}$. The thermocouples at the inlet of each stream and the outlet of the two streams are arranged as shown in figure 4.

The air mass flow rate of the hot and cold air streams are measured by two standard sharp edged ( $d$ and $\mathrm{d} / 2$ tapping) orifice plates of $35 \mathrm{~mm}$ throat diameter and the ratio between orifice diameter and pipe diameter equals 0.33 . The hot air orifice is placed before the heater.

A vertical U-tube manometer is used to measure the pressure difference across the cold stream by balancing the weight of a water column between the first heat recovery section HR1 and the last HR section HR3.

\section{Movable Metal Tube Holding T/C}




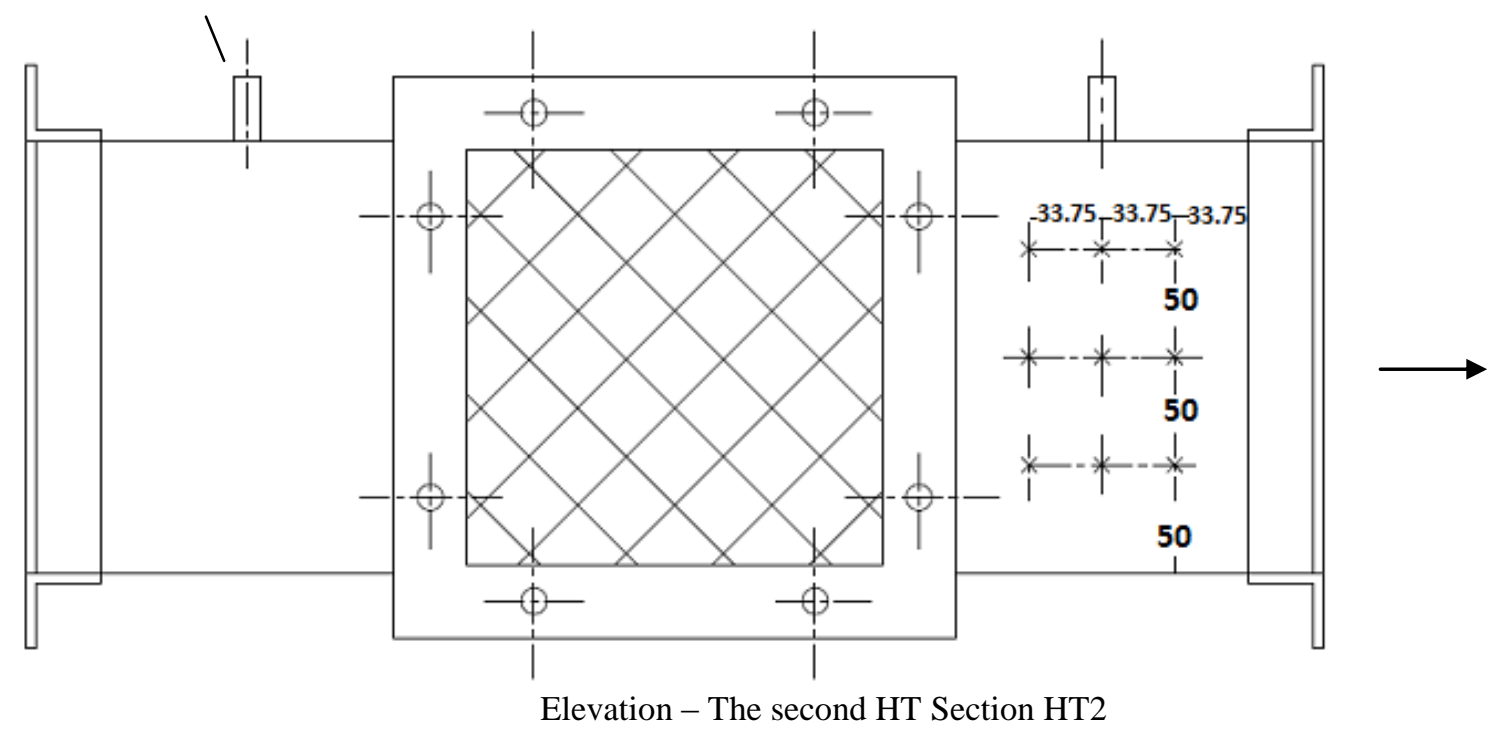

Fig. (4) Location of Temperature Measurements (all dimensions in mm)

\section{OPERATING CONDITIONS}

Experiments were carried out using $(200 \times 200 \mathrm{~mm}$ square $)$ porous material segments whose thicknesses were $30 \mathrm{~mm}, 50 \mathrm{~mm}$ and $70 \mathrm{~mm}$. Experiments were also carried out in the absence of porous media $(0 \mathrm{~mm})$. The key parameters in the present experiments are selected as follows; the hot air inlet temperature was varied using the electric heater. Runs were made at hot air inlet temperatures of $100,200,300,400$ and $500{ }^{\circ} \mathrm{C}$. In each run air this volume flow rates for the hot and cold streams were equal. Experimental runs were carried on for flow rates $0.2,0.25,0.3,0.35,0.4,0.45$ and 0.5 $\mathrm{m}^{3} / \mathrm{min}$. In this paper the results of the extreme and medium flow rates of $0.2,0.35$ and $0.5 \mathrm{~m}^{3} / \mathrm{min}$. only are presented for space limitations. These conditions to flow Reynolds numbers 1040, 1820 and 2600 based on the hydraulic diameters $0.2 \mathrm{~m}$ and inlet temperature $30{ }^{\circ} \mathrm{C}$. The cold air temperature during these runs was nearly constant at $29^{\circ} \mathrm{C}$. 


\section{The Method of Thermo-Hydraulic Performance Analysis}

The thermal performance of the heat exchanger in this work is determined by measuring the hot and cold streams inlet and exit temperatures from the heat exchanger under the different operation conditions.

The heat recovery rate which is the net heat gained by the cold air as it flows through the heat exchanger is calculated by equation 1 .

$$
Q_{c}=\dot{m}_{c^{*}} \cdot C_{p, c^{*}}\left(T_{c_{i, 0}}-T_{c_{i, i}}\right)
$$

The net enthalpy lost from the hot air as it flows through the heat exchanger is given by equation 2

$$
Q_{h}=\dot{m}_{h} \cdot C_{p, h} \cdot\left(T_{h, i}-T_{h_{i} O}\right)
$$

The heat exchanger will lose some of heat to the colder surroundings from both of the hot air and the cold air streams as they flow through the heat exchanger chambers and the piping. The rate of heat loss depends upon the insulation material and thickness. Thus, a reduction of the heat recovery rate $Q_{c}$ than the enthalpy drop of the hot air stream $Q_{h}$ is expected. The percentage loss of the enthalpy from both of the air streams is given by equation 3

$$
\% Q_{\text {loss }}=\frac{Q_{h}-Q_{c}}{Q_{n}} \times 100
$$

The performance of the type of the heat exchanger under consideration in this work is usually evaluated in terms of the heat recovery ratio $\mathrm{H}_{\mathrm{R}}$ defined by

$$
H_{R}=\frac{\dot{m}_{c} \times c_{p, c} \times\left(T_{c, 0}-T_{c_{i}, i}\right)}{\dot{m}_{h} \times c_{p, i} \times\left(T_{h_{i} i}-T_{c_{i} i}\right)}
$$

The heat recovery ratio $\mathrm{H}_{\mathrm{R}}$ as defined by equation (4) is adopted also in the present work for this purpose. This value of the heat recovery ratio is equal to the heat exchanger effectiveness as the mass flow rates of both of the streams are equal and no variation of the air specific heat with temperature is considered.

The pressure drop along the cold air flow pass - from the entry to the first heat recovery chamber till the exit from the bottom low temperature chamber which is the longest passage having the longest connecting piping - represents the hydraulic performance of this heat exchanger.

\section{The Results and Discussions}

Experiments in the procedure described above were undertaken without and with porous inserts of different thicknesses at various air volume flow rates and several hot air temperatures while the cold air temperature was kept nearly constant and the internal surfaces of the heat exchanger chambers are bare without fins. The results using finned internal walls- with and without the insertion of porous segments in the gas paths is the subject of a following paper. The thermal-hydrodynamic performance of this five chamber heat exchanger and its comparison with that of three chambers one is presented in this section.

\subsection{Cold Air Outlet Temperature}

The temperature of the cold air leaving the heat exchanger is one of its most important performance characteristics as it determines the class of application at which it can be used. The effects of the hot air inlet temperature to the high temperature chamber $T_{h, i}$ and the porous media thickness on the cold air outlet temperature Tc,o at different air volume flow rates for the present five layered heat exchanger are shown in figures 5,6 and 7. On the same figures the results previously obtained for this temperature using the three layered heat exchanger under the same operating conditions abstracted from reference $[1]$ is displayed on the same figures. 


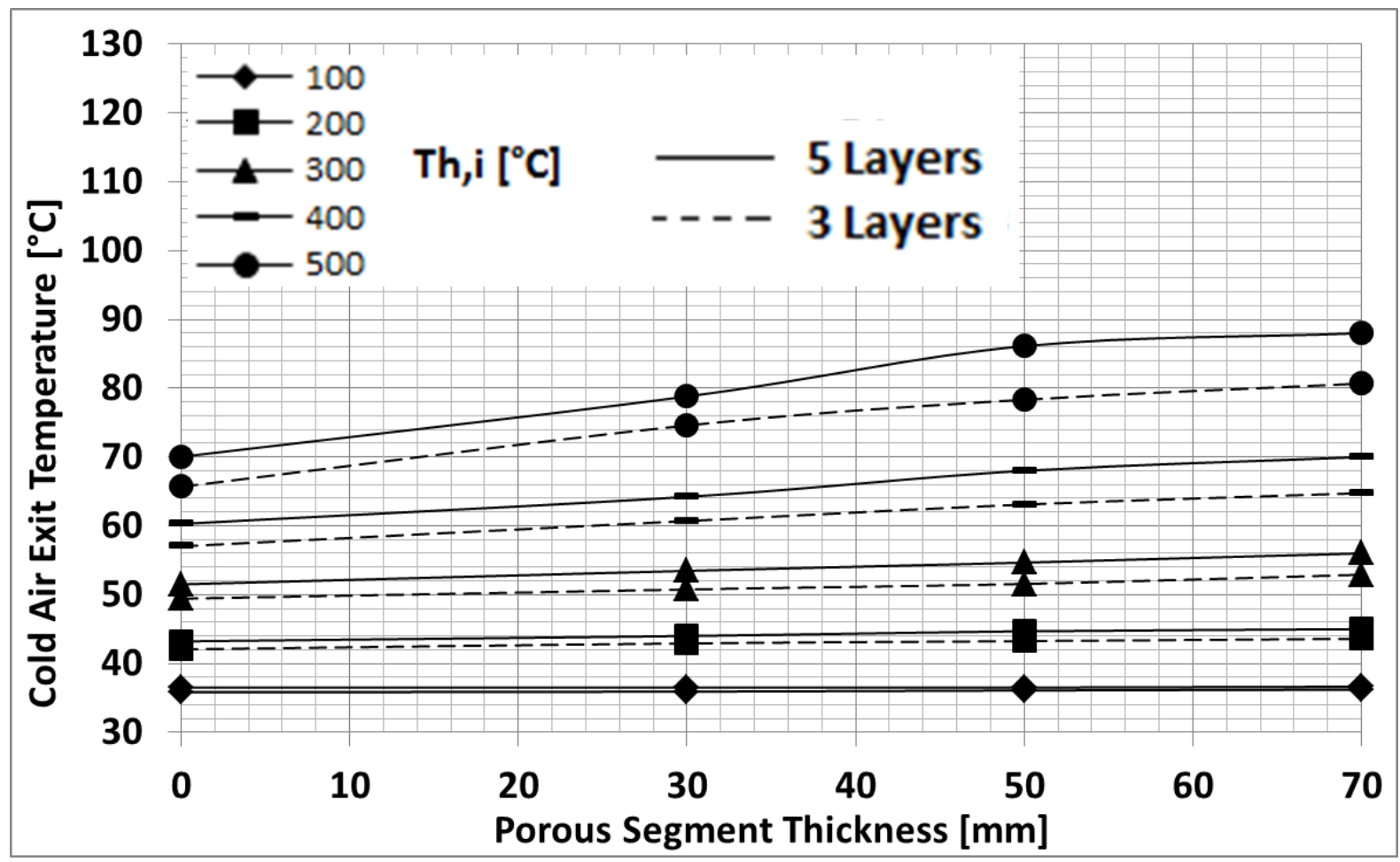

Fig. (5) Effects of $T_{h, i}$ and porous media thickness on $T_{c, 0}$ at volume flow rate of $0.2 \mathrm{~m}^{3} / \mathrm{min}$. It is observed that at the lowest hot air inlet temperature $100{ }^{\circ} \mathrm{C}$, the cold air outlet temperature increases with the increase of the porous segment thickness at an insignificant rate to the extent that this temperature seems to be nearly constant at different volume flow rates. Also, from these figures, it is obvious that at the hot air inlet temperatures of $200{ }^{\circ} \mathrm{C}$ and $300^{\circ} \mathrm{C}$, the cold air temperature is increasing almost linearly with the increase of the porous segments thickness but also at a low rate.

At the high hot air inlet temperatures of $400^{\circ} \mathrm{C}$ and $500^{\circ} \mathrm{C}$, the cold air temperature is increasing rapidly with the increase of the porous segment thickness up to $50 \mathrm{~mm}$. At a thickness of about $50 \mathrm{~mm}$ and more the cold air temperature increases at a lower rate approaching an asymptotic value. This behavior shows up very clearly at higher volume flow rates as shown in figures 6 and 7 . At the same operation conditions, the cold air temperature is increasing in the five layered heat exchanger than that of the three layered one. When the flow rate of both of the hot and cold streams are $0.5 \mathrm{~m}^{3} / \mathrm{min}$, the cold air outlet temperature reaches $123.3^{\circ} \mathrm{C}$ in the five layered heat exchanger while it was only $108.75^{\circ} \mathrm{C}$ in the three layered one using porous segments of $70 \mathrm{~mm}$ thickness at the hot air inlet temperature of $500^{\circ} \mathrm{C}$ giving 13.4 percent increase in the exit temperature and 37 percent corresponding increase in the cold air stream pressure drop as demonstrated in figure 20 . 


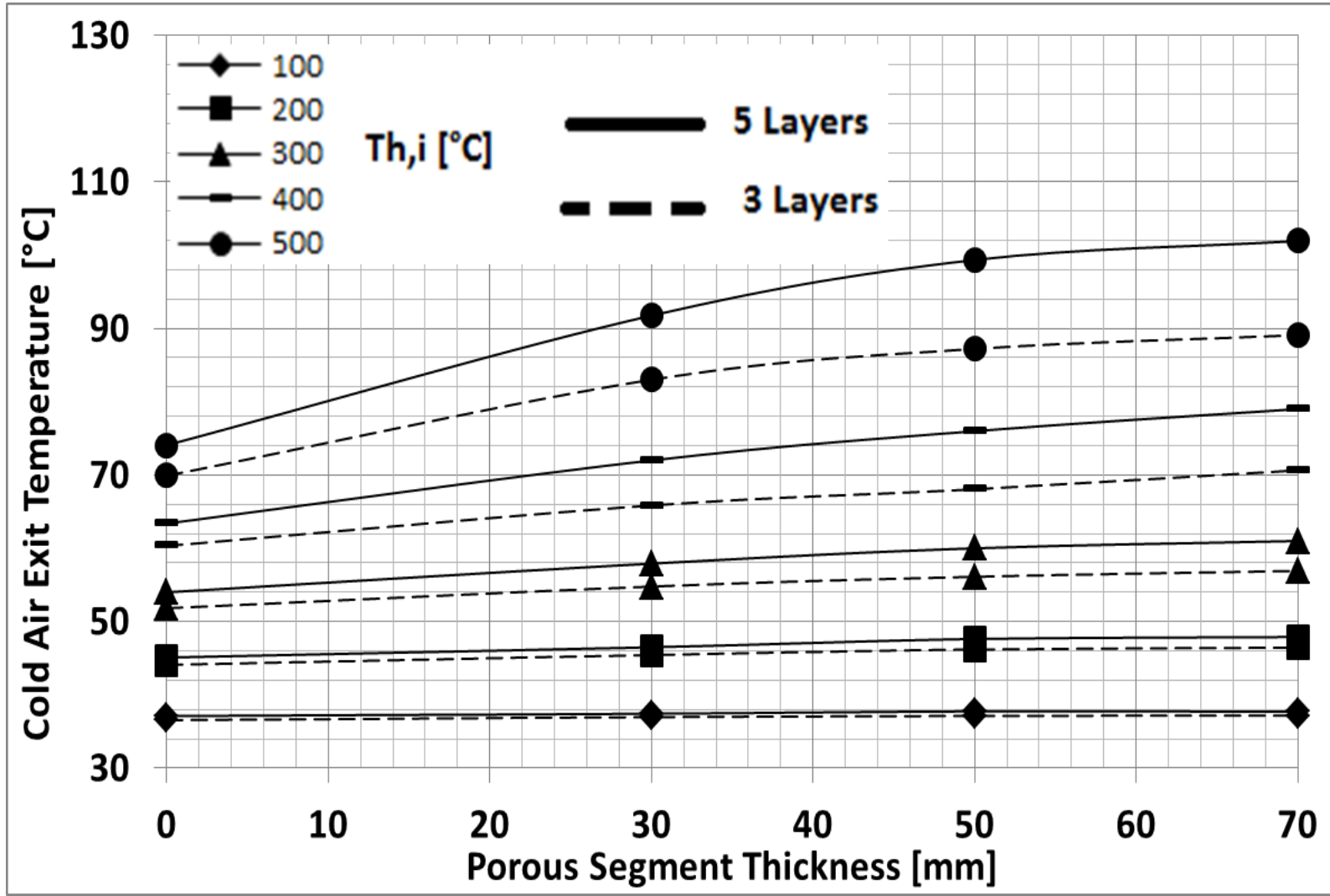

Fig. (6) Effects of $T_{h, i}$ and porous media thickness on $T_{c, 0}$ at volume flow rate of $0.35 \mathrm{~m}^{3} / \mathrm{min}$.

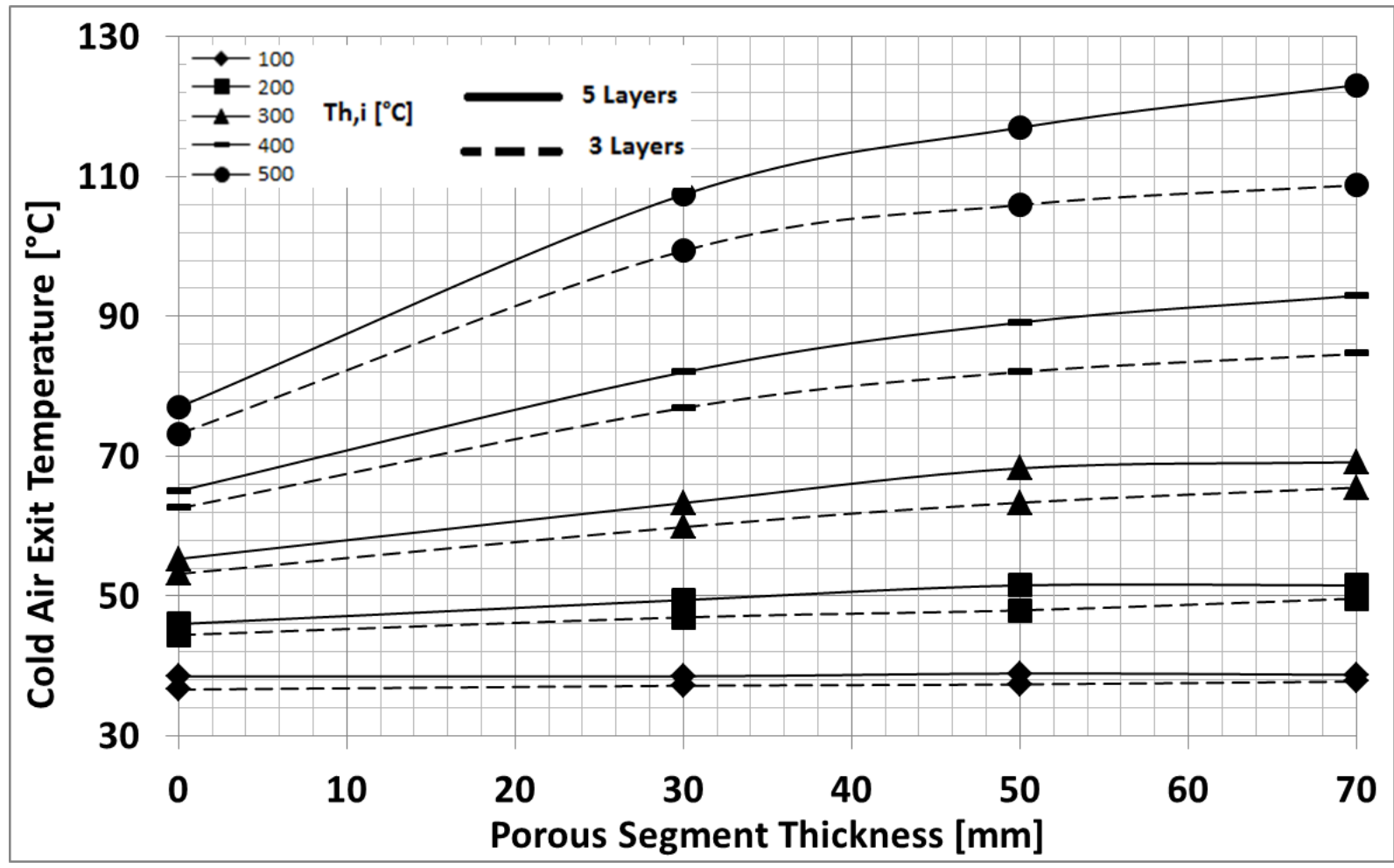

Fig. (7) Effects of $T_{h, i}$ and porous media thickness on $T_{c, 0}$ at volume flow rate of $0.5 \mathrm{~m}^{3} / \mathrm{min}$. 


\subsection{Hot Air Outlet Temperature}

The heat transfer takes place through the separating walls in the manner described before from the hot air to the cold air as each of them flows in its own passage. The hot air outlet temperature decrease varies with the porous material thickness, the hot air inlet temperature, and the two air volume flow rates. The number of the heat exchanger layers is another effective parameter. The change in the hot air outlet temperature with the porous material thickness at different values of the hot air inlet temperatures and air volume flow rates is plotted in figures 8,9 and 10 .

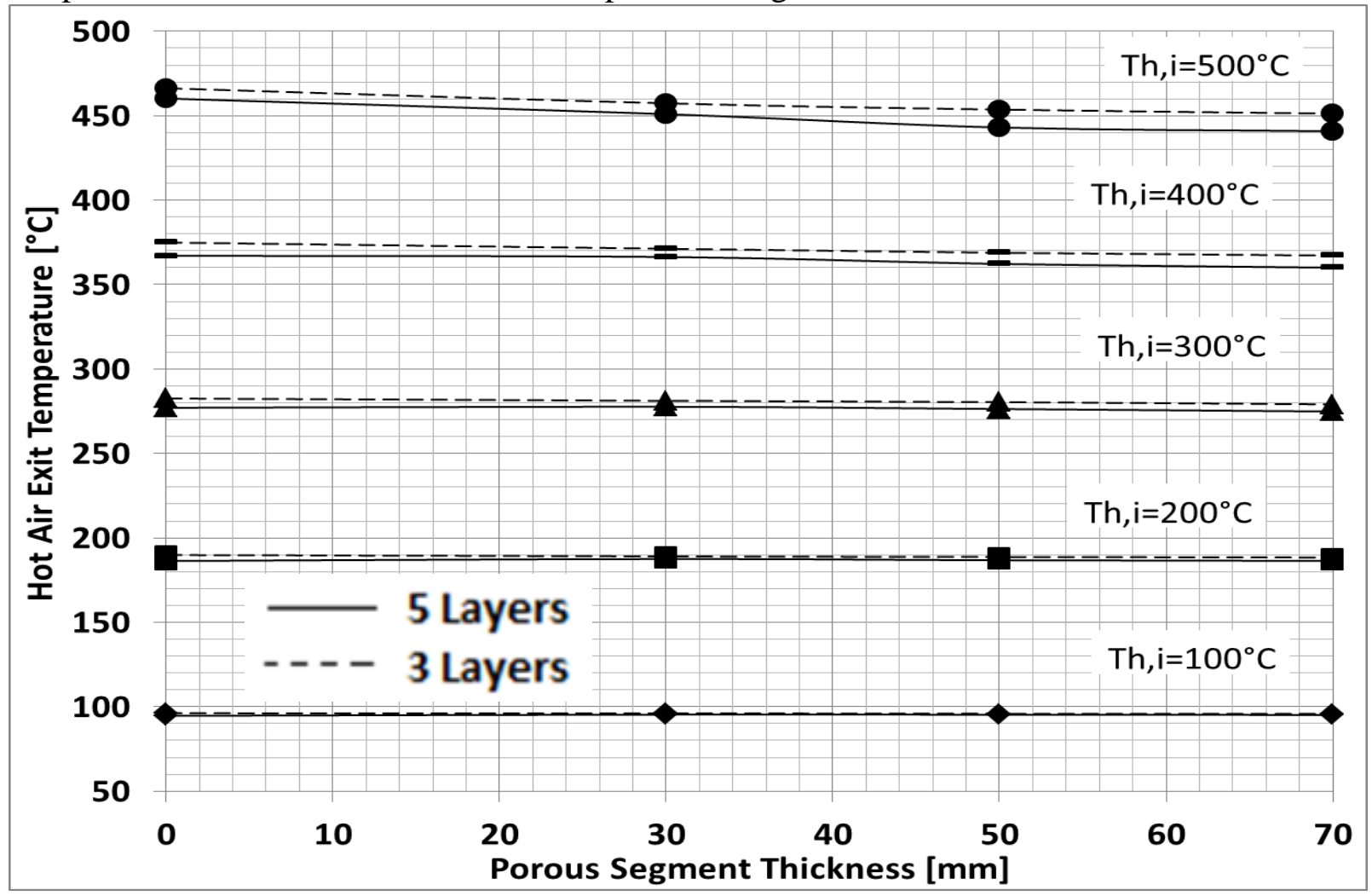

Fig. (8) Effects of $T_{h, i}$ and porous media thickness on $T_{h, o}$ at volume flow rate of $0.2 \mathrm{~m}^{3} / \mathrm{min}$.

As the porous media thickness increases the hot air outlet temperature starts to decrease as the hot air exchange more heat with the porous material by convection that is exchanged by radiation with the interior surfaces of the hot chambers and is ultimately transferred to the cold air. As shown in the figures, the hot air exit temperature seems as if it is constant at the low hot air inlet temperature of $100^{\circ} \mathrm{C}$ at the different volume flow rates because of the low convective heat transfer coefficients on both sides of the separating walls, the lowest hot-cold air temperature difference and the low emissive power of the porous material and the interior surrounding surfaces of the hot air chambers. While at the hot air inlet temperatures of $200^{\circ} \mathrm{C}, 300^{\circ} \mathrm{C}$, and $400^{\circ} \mathrm{C}$ the decrease in the hot air outlet temperature becomes more pronounced at the high air volume flow rates because the above mentioned heat transfer parameter attain somewhat higher values but retains the linear relationship with the porous segment thickness. 


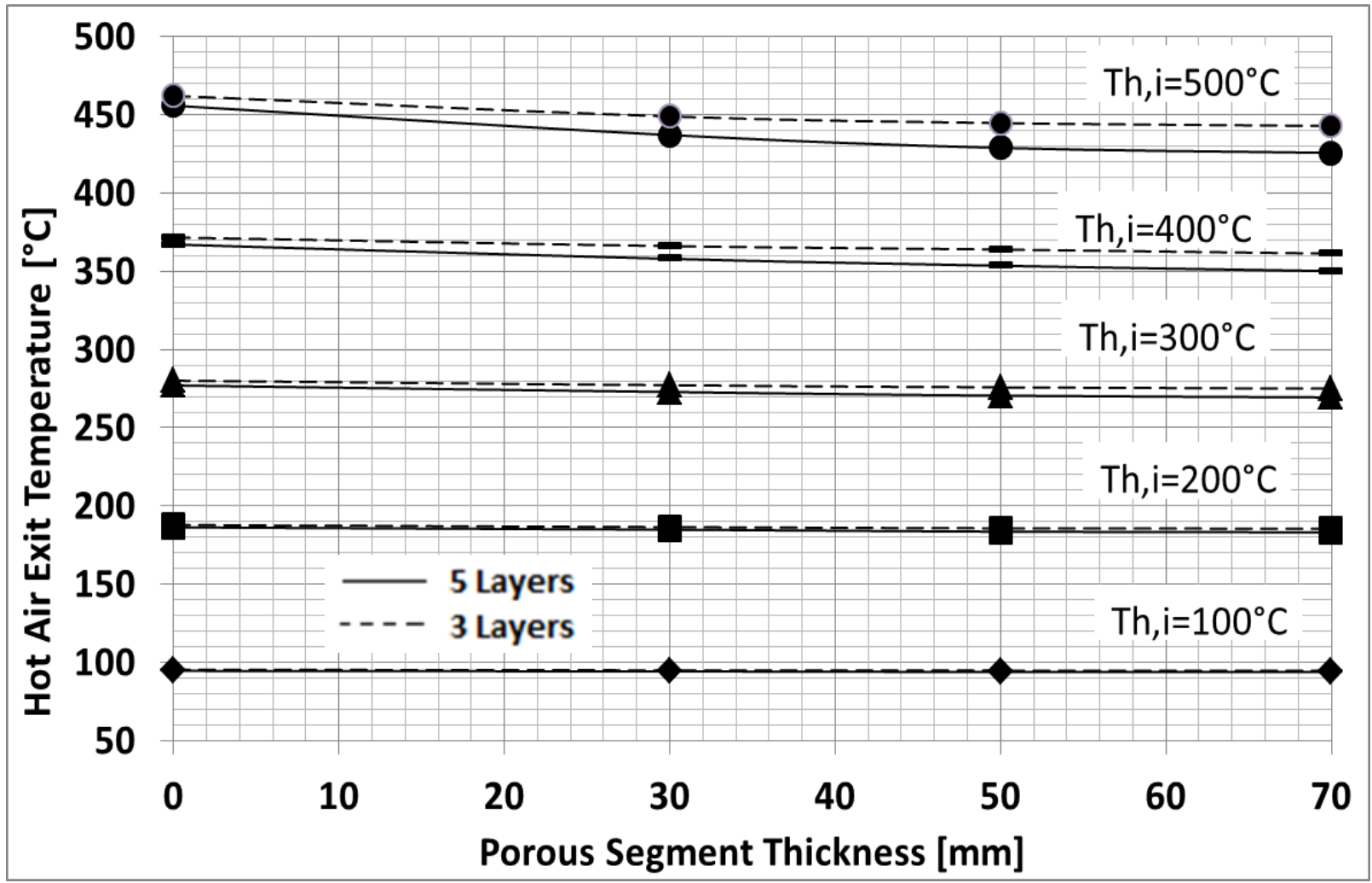

Fig. (9) Effects of $T_{h, i}$ and porous media thickness on $T_{h, o}$ at volume flow rate of $0.35 \mathrm{~m}^{3} / \mathrm{min}$.

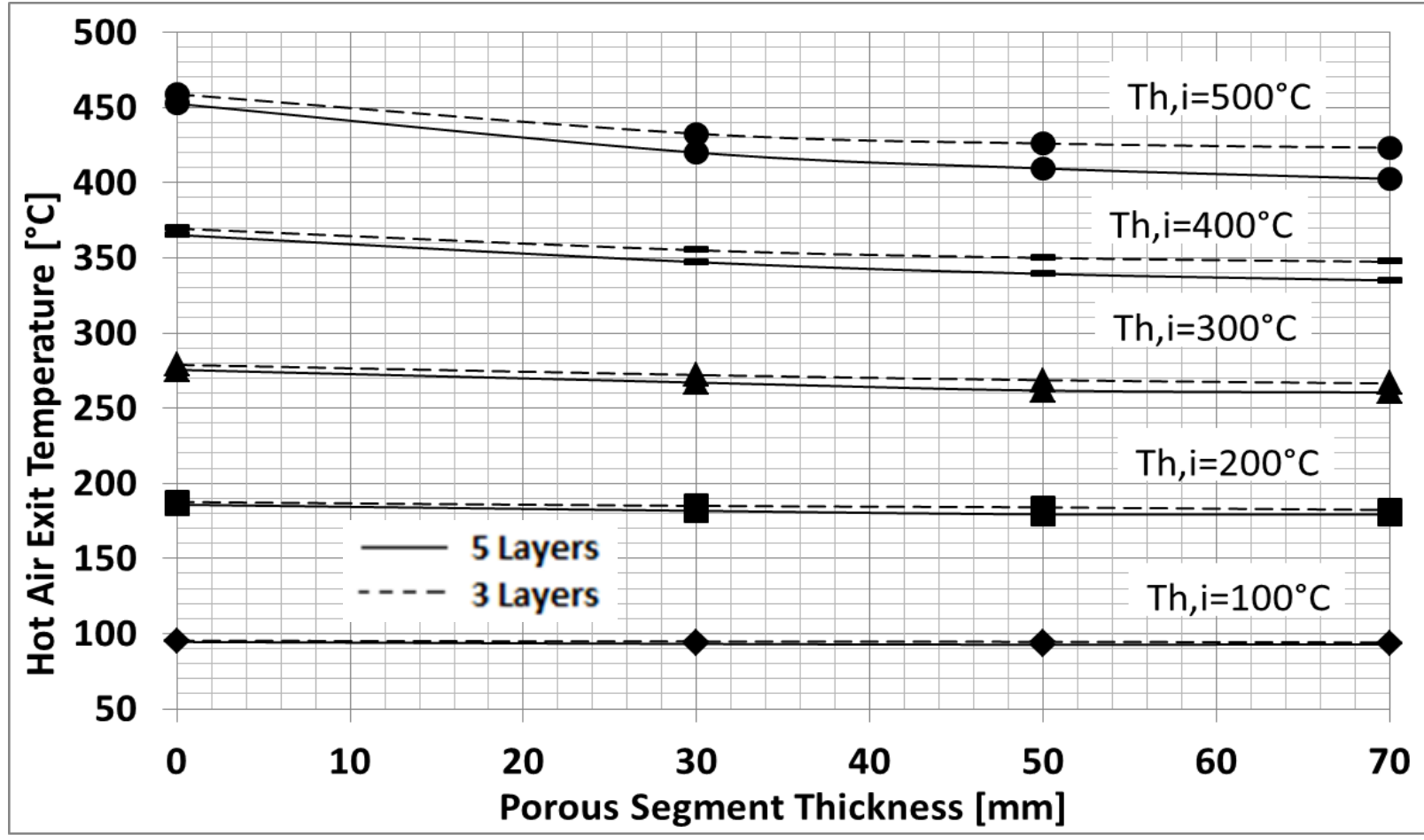

Fig. (10) Effects of $T_{h, i}$ and porous media thickness on $T_{h, o}$ at volume flow rate of $0.5 \mathrm{~m}^{3} / \mathrm{min}$ 
Figures 8, 9 and 10 also indicate that at higher hot air inlet temperature level $500^{\circ} \mathrm{C}$, the hot air outlet temperature decreases rapidly with the porous media thickness up to a porous medium thickness of 50 $\mathrm{mm}$ then its rate of change decreases as the porous material thickness increases beyond $50 \mathrm{~mm}$. It is also clear, that at any porous material thickness and a hot air inlet temperature by increasing the air volume flow rate, the hot air outlet temperature decreases.

The results presented in this section show that at the largest porous segment thickness used in the three layered heat exchanger, $70 \mathrm{~mm}$, causes some insignificant decrease in the outlet hot air temperature and the hot gases exit temperature levels are still high. At the hot air inlet temperature of $500^{\circ} \mathrm{C}$, air volume flow rate $0.5 \mathrm{~m}^{3} / \mathrm{min}$ and at porous medium thickness of $70 \mathrm{~mm}$ the hot gases leaves the three layered heat exchanger at $419.5^{\circ} \mathrm{C}$ while it leaves the five layered one at a lower temperature $405.6^{\circ} \mathrm{C}$. The large difference between the high level of the hot gas exit temperature which is obvious in figures 8, 9 and 10 and the low temperature levels of the cold air exit temperature shown in figures 5, 6 and 7 provides a high driving potential difference enough for additional heat transfer between the hot and cold streams. For this reason the five layered heat exchanger, providing an extra heat exchange chamber for each of the hot and cold air stream helps to decrease the hot air outlet temperature to lower values than that of the three layered one at the same operation conditions. It also increases the cold air exit temperature of the five chamber heat exchanger than that of the three chambers one.

Addition of fins contribute to the increase of the heat exchange driving mechanism in the five layered heat exchanger as it was the case of the three layered heat exchanger given in reference 1, which is the subject of a following paper.

It is noticed that the mode of change of the hot air outlet temperature with the porous material thickness is a reflection of that of the cold air. At the low inlet temperature of the hot gas it showed insignificant change, at the intermediate temperature they also vary linearly with the porous material thickness undergoing low temperature rise at all volume flow rates. At the high hot air temperature, these intrareflection are the same for the both streams at all volume flow rates.

\subsection{The Heat Recovery Ratio}

The heat recovery ratio which represents the effectiveness of this heat exchanger was calculated from equation 4. The effects of the porous segment thicknesses, the air volume flow rate, and the hot air inlet temperature on the heat recovery ratio are examined and are presented in figures 11, 12 and 13. From these figures, it is clear that by increasing the hot air inlet temperature the heat recovery ratio increases. Also this increase becomes more by using porous segments of higher thicknesses. The figures are showing that at lower hot air inlet temperatures of $100^{\circ} \mathrm{C}, 200^{\circ} \mathrm{C}$, and $300^{\circ} \mathrm{C}$; the heat recovery ratio increases almost linearly with the increase of the porous medium thickness. As the temperature level of the hot air inlet temperature becomes higher, the heat recovery ratio rises more rapidly. With increasing the thickness to about $50 \mathrm{~mm}$, the heat recovery ratio undergoes a slight increase to the extent that it can be considered almost constant at higher hot air inlet temperatures of 400 and $500^{\circ} \mathrm{C}$. These results show that the role of the previously described energy conversion mechanism starts to be more effective at the high temperatures with the increase of the porous material thickness up to $50 \mathrm{~mm}$. As the temperature increases the enthalpy of the high temperature gas is effectively transferred to the porous material plate via an efficient convective heat transfer process. Under steady state operation the amount of energy transferred from the high temperature gas to the porous segment is emitted to the enclosing walls by the porous plate that acquired a stronger emissive power.

These results also indicate the effect of the structure of the heat exchanger, the five layer heat exchanger is more effective than the three layered at the same operating condition. At the hot air inlet temperature of $500^{\circ} \mathrm{C}$, air volume flow rate $0.5 \mathrm{~m}^{3} / \mathrm{min}$ and using a porous material of $70 \mathrm{~mm}$ thickness the heat recovery ratios is 0.195 in the present five layered heat exchanger while this value was 0.164 in the three layered one. The results obtained in this work, that is subject of a following paper, showed that addition of fins helps to considerably improve the effectiveness of this heat exchanger as it is also the case in the three layer one [1]. 


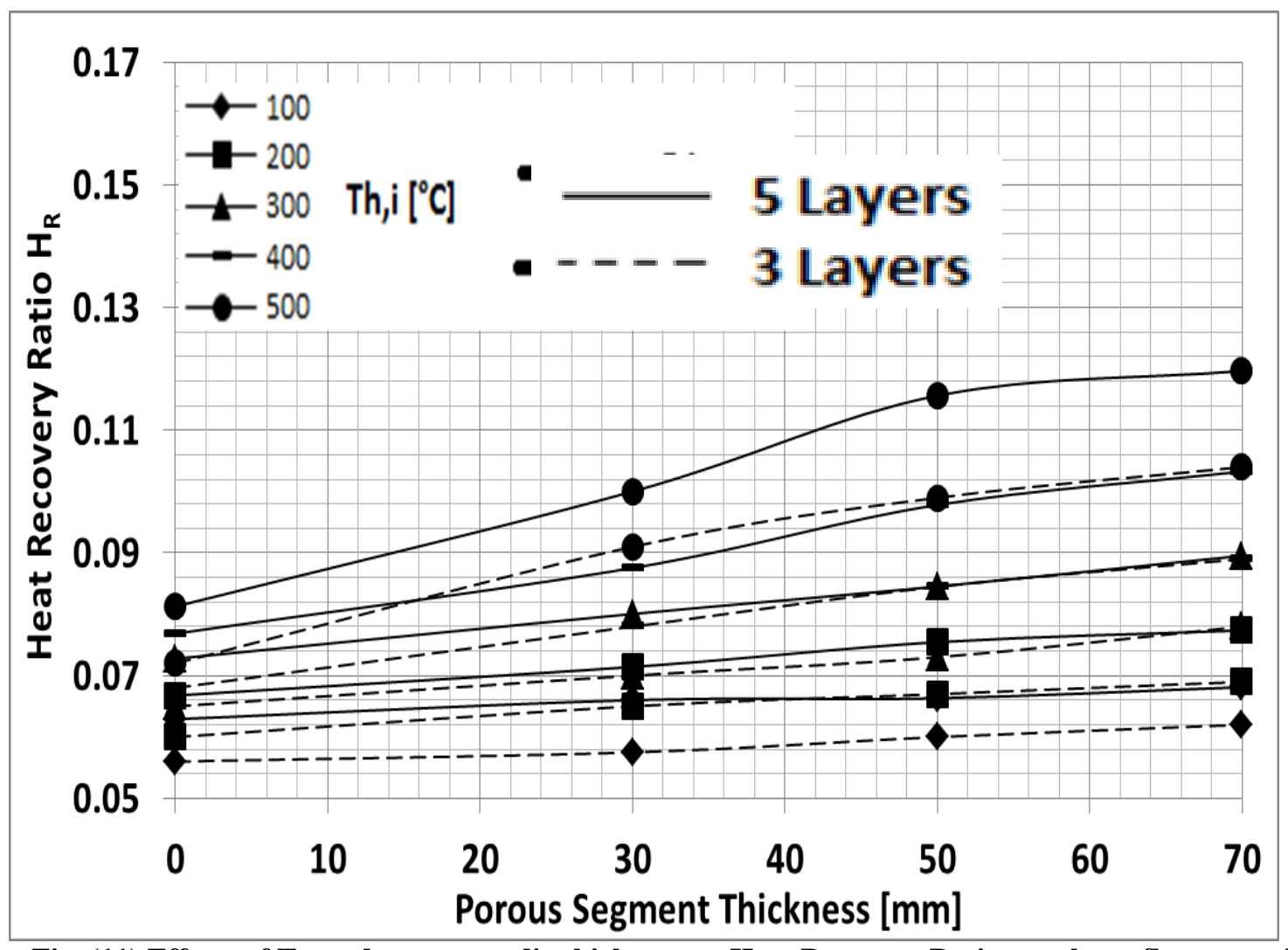

Fig. (11) Effects of $T_{h, i}$ and porous media thickness on Heat Recovery Ratio at volume flow rate of 0.2 $\mathrm{m} 3 / \mathrm{min}$.

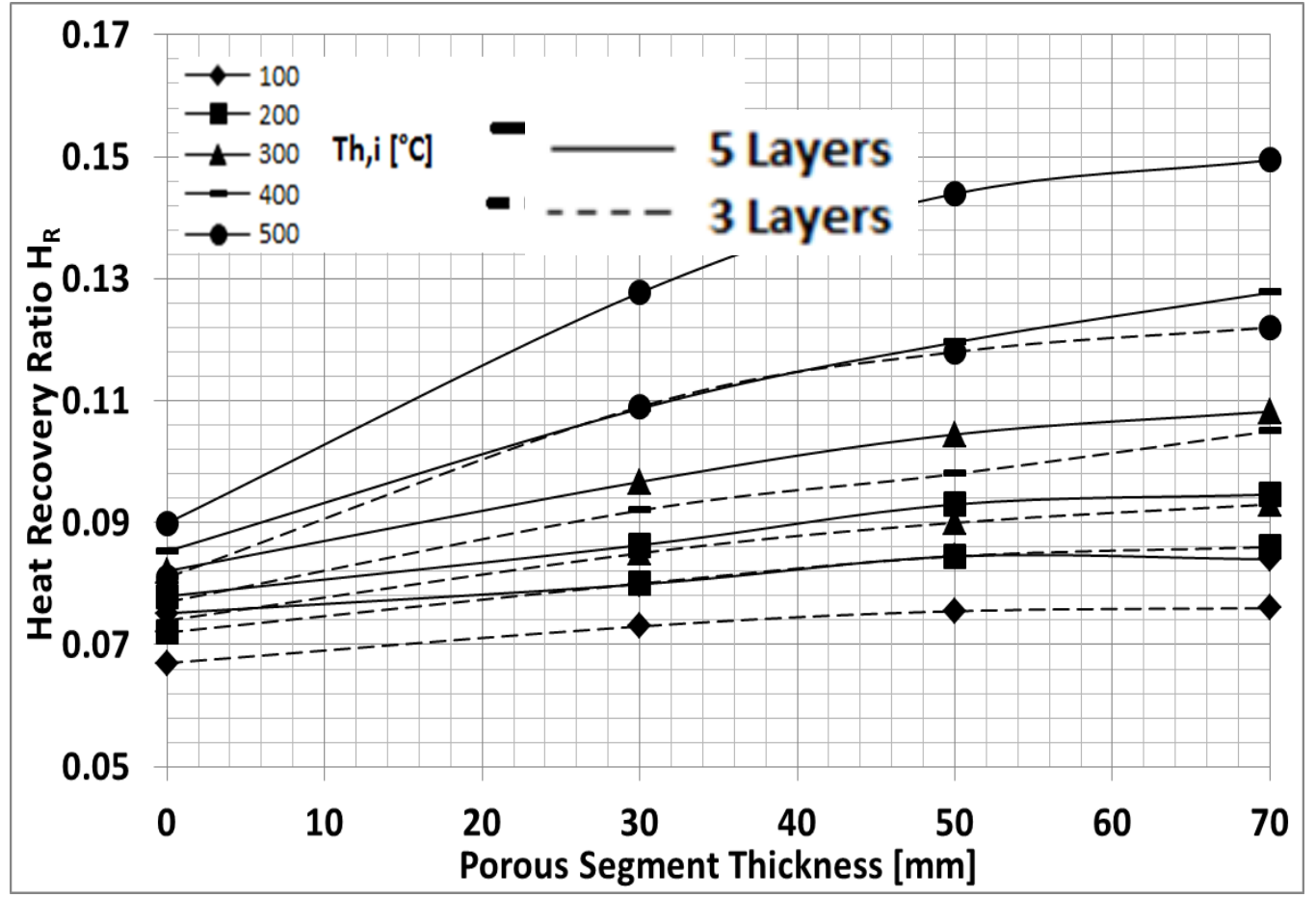

Fig. (12) Effects of $T_{h, i}$ and porous media thickness on Heat Recovery Ratio at volume flow rate of 0.35 $\mathrm{m3} / \mathrm{min}$. 


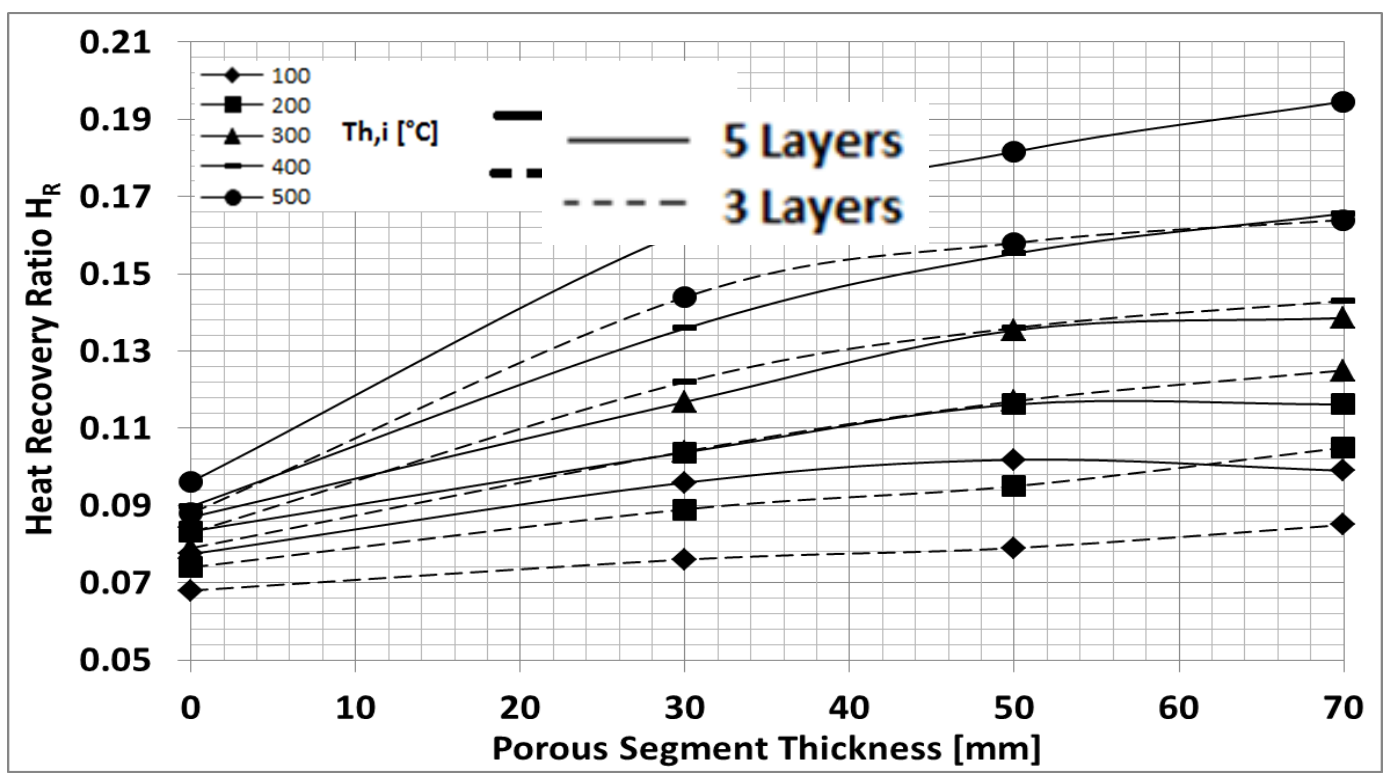

Fig. (13) Effects of $T_{h, i}$ and porous media thickness on Heat Recovery Ratio at volume flow rate of 0.5 $\mathrm{m} 3 / \mathrm{min}$.

\subsection{The Heat Recovery Rate}

From equation 2, the heat recovery rate $Q_{c}$ was calculated and are plotted in figures 14,15 and 16 to illustrate the impact of each of the hot air inlet temperature $\mathrm{T}_{\mathrm{hi}}$, the air streams volume flow rate and the porous material thickness on the heat recovery rate for the present five layered heat exchanger.

The results obtained previously in reference [1] are also plotted in these figures for the sake of demonstrating the influence of the number of heat exchange chambers.

Comparison of the results shown in these figures shows that using the five layered heat exchanger increases the heat recovery rate over that would be obtained using the three layered one at the same operating conditions.

It is shown in these figures that the heat recovery rate increases with the increase of the porous material thickness. Furthermore, the results in these figures reflect the changes in each of the cold air and the hot air exit temperatures as well as the heat recovery ratio discussed above. It is observed that at hot air inlet temperature of $100^{\circ} \mathrm{C}$ the rise in the heat recovery rate with the increase of porous material thickness is almost negligible at the different volume flow rates. At hot air inlet temperatures of 200, 300 , and $400^{\circ} \mathrm{C}$ the rate of increase of the heat gained is almost linear with a slope increasing by the increase of the hot air inlet temperatures. At the high hot air inlet temperature of $500^{\circ} \mathrm{C}$ the heat gained increases rapidly by increasing the porous material thickness till a thickness of $50 \mathrm{~mm}$, beyond which the heat recovery rate tends to go to an asymptotic value as shown in the figures.

These results show that the increase of the volume flow rate of the two air streams at any hot air temperature and any porous insert thickness results in an increase in the heat recovery rate due to the increase of the convective heat transfer coefficients from the fluids to the porous segments and the exterior walls of the chambers as well since they also contribute to the hot-cold streams separating walls. 


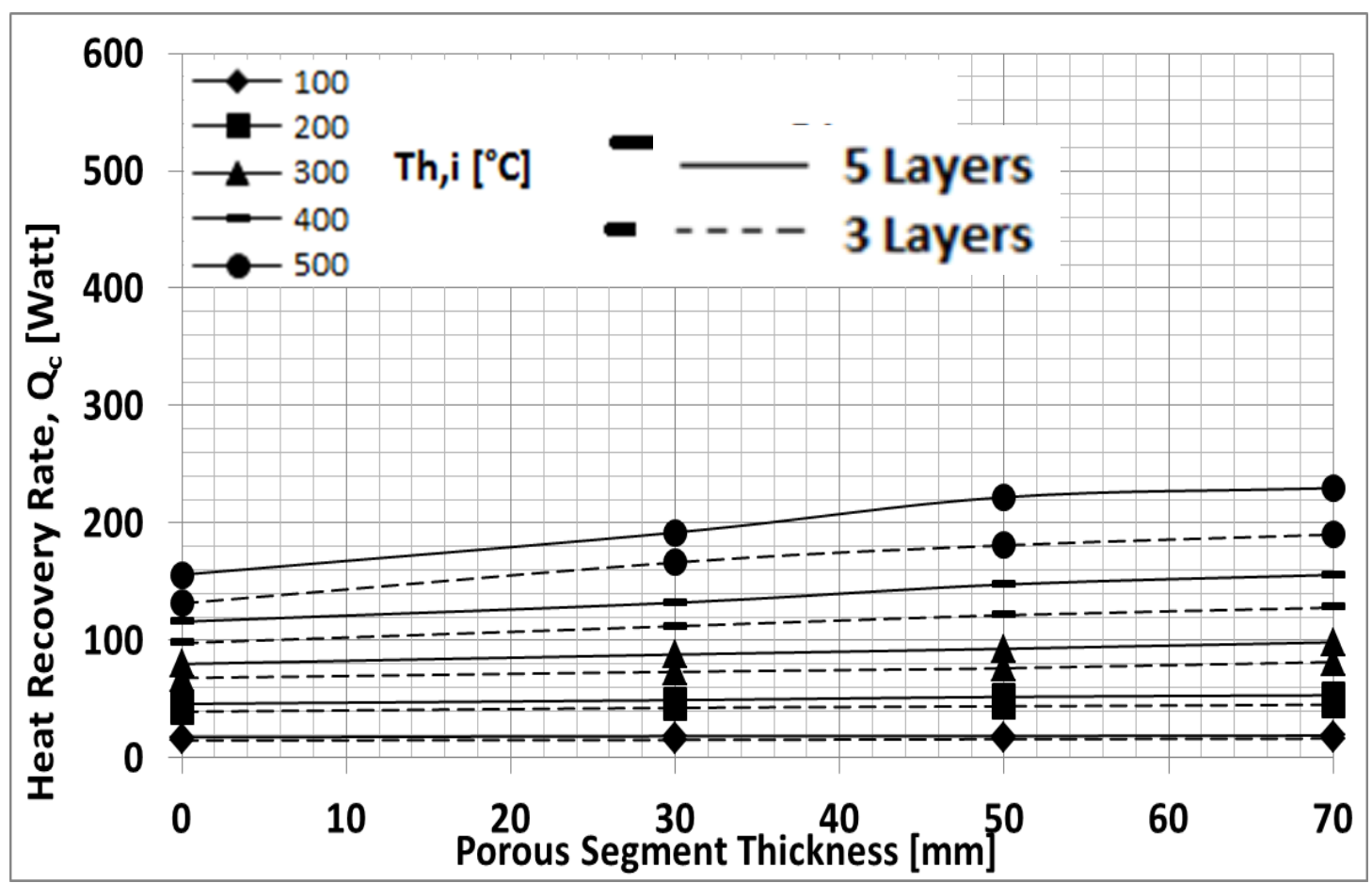

Fig. (14) Effects of $T_{h, i}$ and porous media thickness on Heat Recovery Rate at volume flow rate of 0.2 $\mathrm{m} 3 / \mathrm{min}$

The increase of the convective heat transfer coefficient between the hot air stream and the porous medium on the hot air side caused by the increase of its flow rates rises the steady state temperature of the porous material and its emissive power. In this case, the conversion of the enthalpy of the hot air to radiant energy that ultimately is transferred to the cold air stream through the separating walls increases.

The heat received by the hot air side separating surface through convection and radiation is transferred to the cold air by convection as well as by radiation to the interior surfaces of lower and upper cold air recovery chambers and to the porous inserts in these chambers.

In this case the increase of the heat transfer coefficient between the colder air stream and both of the separating surfaces and the porous inserts on the cold air side due to its higher flow velocities causes a high rate of the convective heat transfer from these surfaces to the colder air flowing through it. This increase of the convective heat transfer coefficient causes an increased conversion of the radiant energy captured by the porous segments in the cold air chambers gained from the radiant energy from the separating and enveloping walls into an increase of the enthalpy of the cold air. In addition, the increase of the convective heat transfer coefficient between the air streams and the hotter enveloping walls, which receive radiant energy from the higher temperature separating walls, shares in further increase of the heat recovery rate by these two modes of heat recovery promotion. 


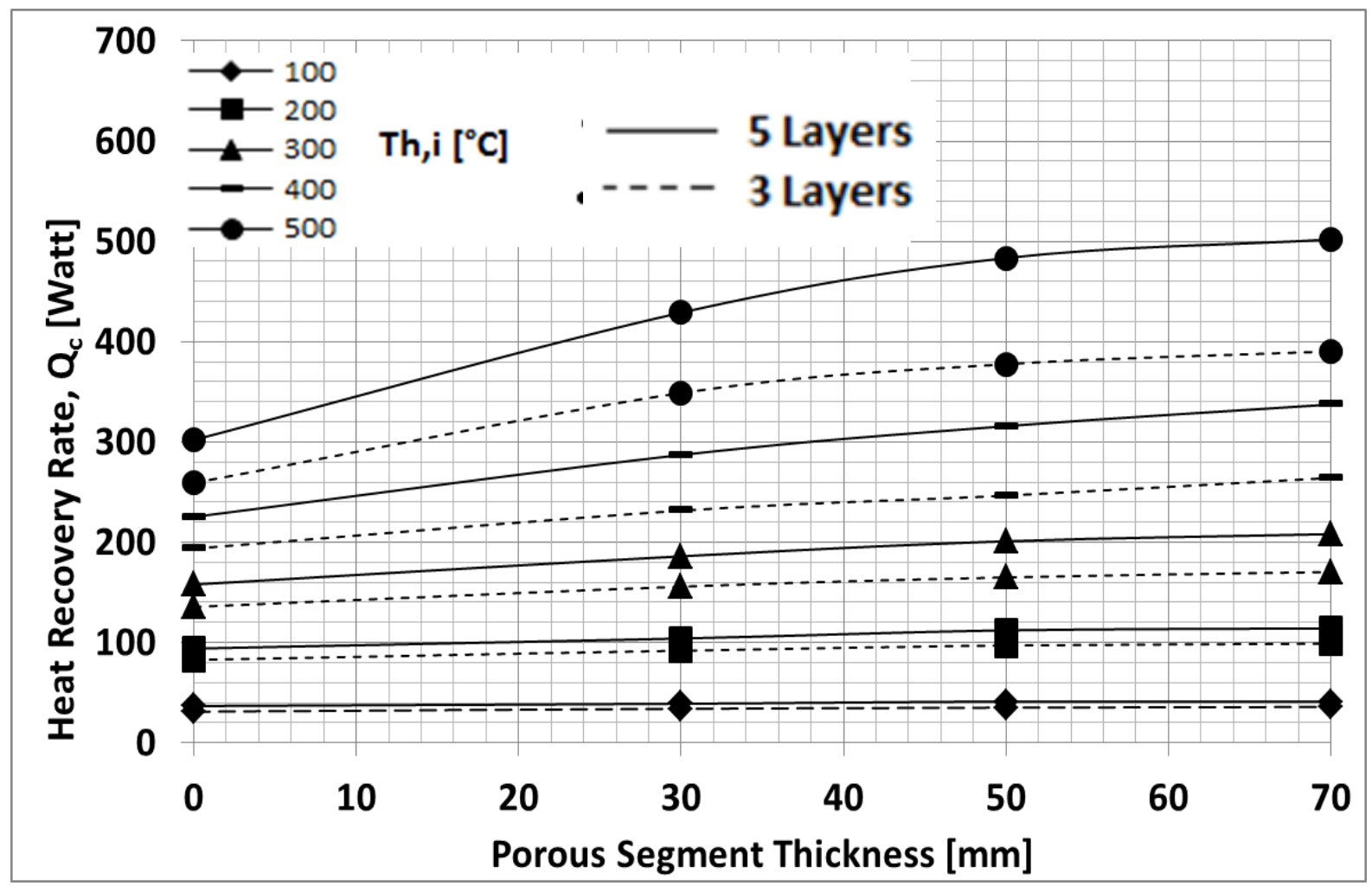

Fig. (15) Effects of $T_{h, i}$ and porous media thickness on Heat Recovery Rate at volume flow rate of $0.35 \mathrm{~m} 3 / \mathrm{min}$.

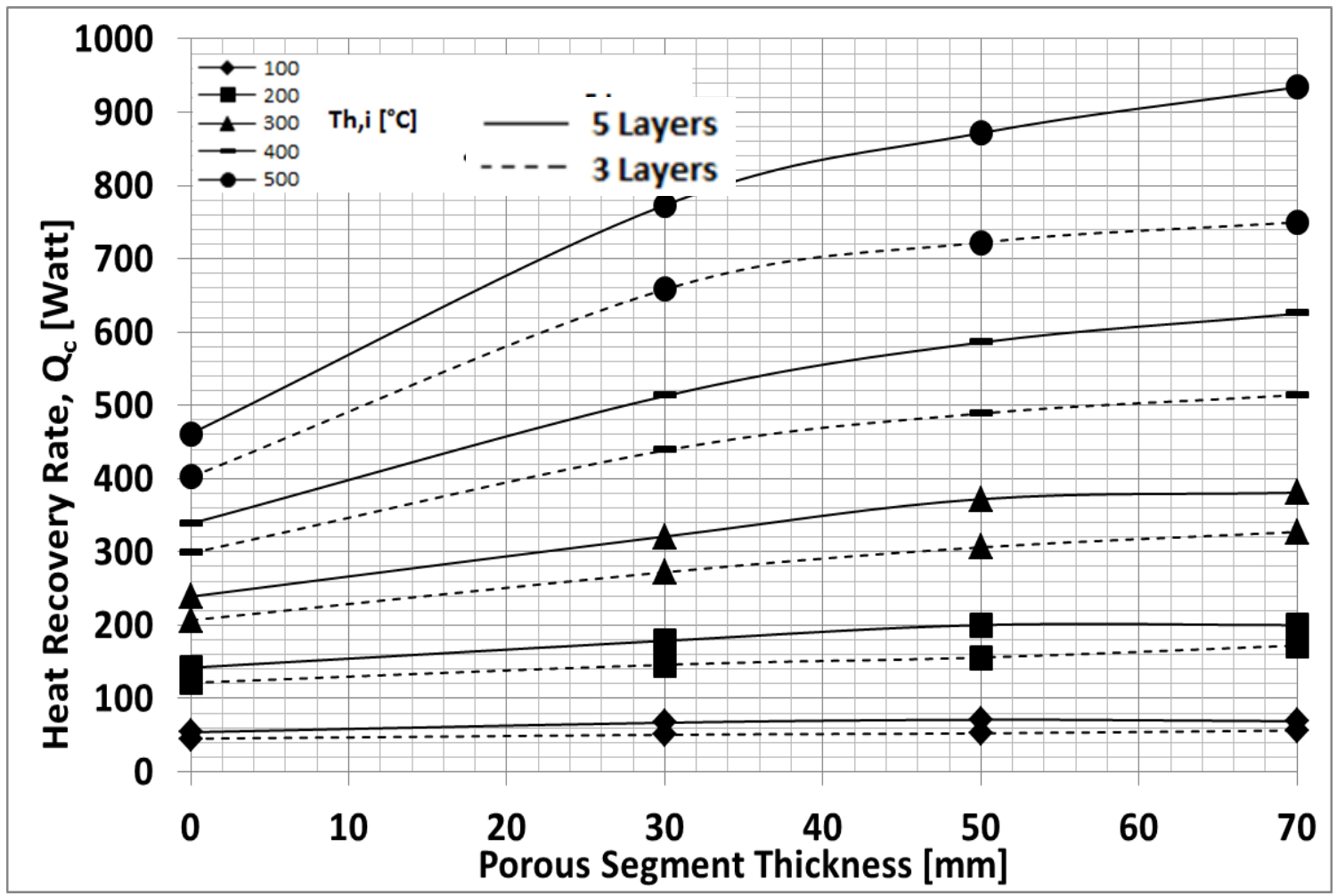

Fig. (16) Effects of $T_{h, i}$ and porous media thickness on Heat Recovery Rate at volume flow rate of 0.5 $\mathrm{m3} / \mathrm{min}$. 
For the three layered heat exchanger, the air volume flow rate of $0.5 \mathrm{~m}^{3} / \mathrm{min}$. and the hot air inlet temperature of $500^{\circ} \mathrm{C}$ the heat recovery rate is 402.3 Watt in the absence of porous material, 658.3 Watt in the case of using porous material of $30 \mathrm{~mm}$ thickness, and 749.7 Watt in the case of using porous material of $70 \mathrm{~mm}$ thickness. The heat recovery rate for the present heat exchanger has increased at the same operation conditions to 474.6 Watt in the absence of porous material, 789.6 Watt in the case of using porous material of $30 \mathrm{~mm}$ thickness, and 936.33 Watt in the case of using porous material of $70 \mathrm{~mm}$ thickness with a corresponding increase of the heat recovery ratio of $18,26.2$ and 18.6 percent respectively. These are favorable rates of increase of the recovery ratios if compared to the pressure loss caused by the insertion of the porous material as discussed in section 7.

\subsection{The Heat Loss Percentages from the Heat Exchanger}

The increase of the number of the chambers of the multi-layered gas to gas heat exchangers augments the heat recovery ratio and the heat recovery rate and also causes an increase in the rate of heat loss. In the present experimental work it was found that the rate of heat loss from the hot gas is higher than that in the three layered exchange, but under the same operating conditions. However the percentage of the heat loss to the drop in the hot air enthalpy in the present case is lower than in the three layered heat exchanger as is determined in figures 17,18 , and 19.

The substation from equations 1 and 2 into equation 3 gives the percentage heat loss for the different operation conditions. The effect of the hot air inlet temperature and the porous media thickness on the heat loss percentage of the considered five layered heat exchanger at different air volume flow rates are shown in figures 17, 18, and 19. The percentage heat loss encountered in the three layered heat exchanger under the same operating conditions are represented in the same figures by the dotted line. For the porous material of $70 \mathrm{~mm}$ thickness at the air volume flow rate of $0.2 \mathrm{~m}^{3} / \mathrm{min}$ for the present heat exchanger the percentage heat loss is 5.4 percent at hot air inlet temperatures of $500^{\circ} \mathrm{C}$, while it is at air volume rate of $0.5 \mathrm{~m}^{3} / \mathrm{min}$ the heat loss is 6.6 percent at hot air inlet temperature of $500^{\circ} \mathrm{C}$ and the porous material of $70 \mathrm{~mm}$ thickness. The percentage of heat loss increased by 21.2 percent due to the increase of the volume flow rate from 0.2 to $0.5 \mathrm{~m}^{3} / \mathrm{min}$. at the same porous material thickness rate and the same hot air inlet temperature. This increase in the percentage of the heat loss is due to the increase of the connective heat transfer coefficient to the insulated surfaces of the two high temperature chambers. 


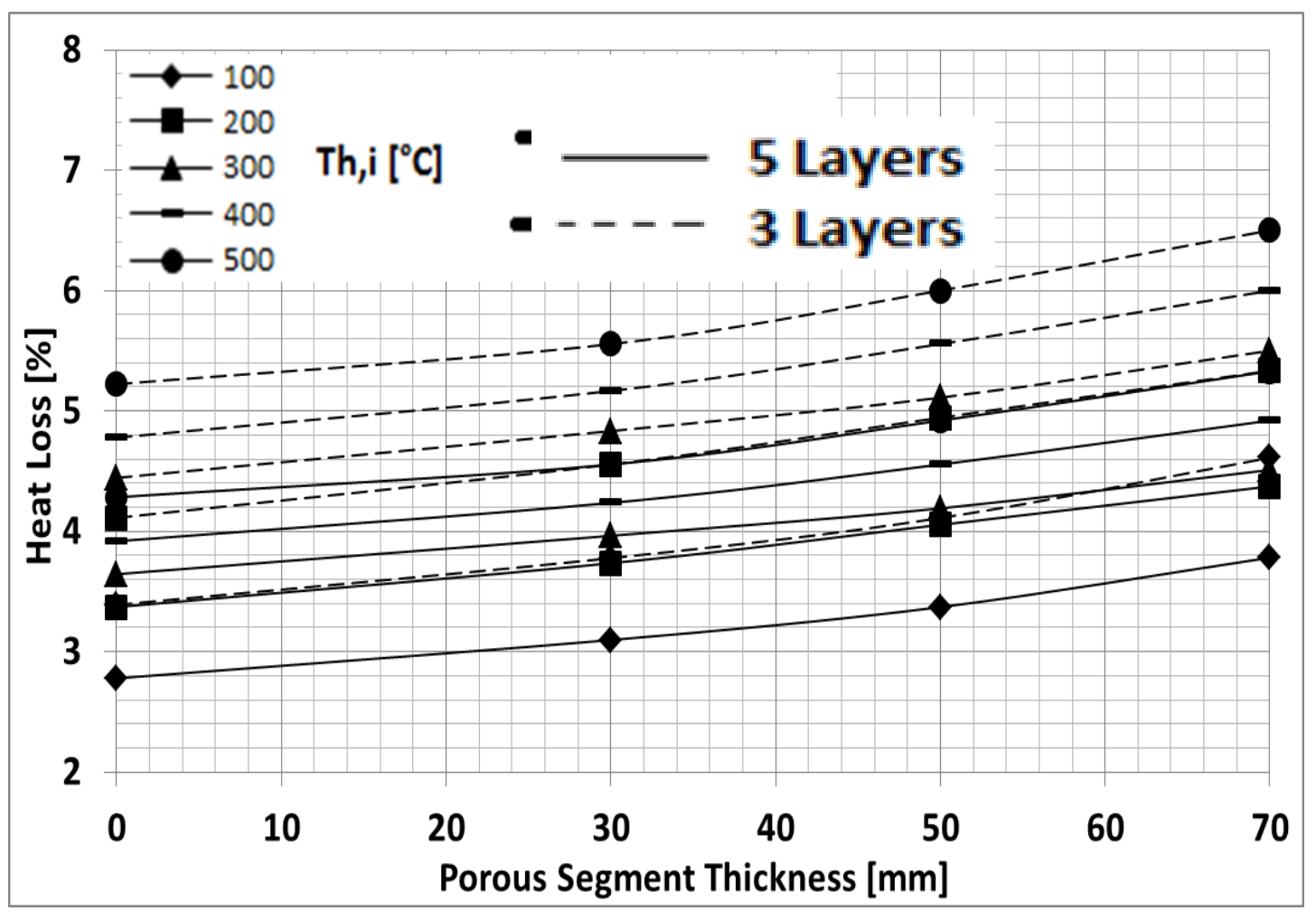

Fig. (17) Effects of $T_{h, i}$ and porous media thickness on Heat Loss at volume flow rate of $0.2 \mathrm{~m}^{3} / \mathrm{min}$.

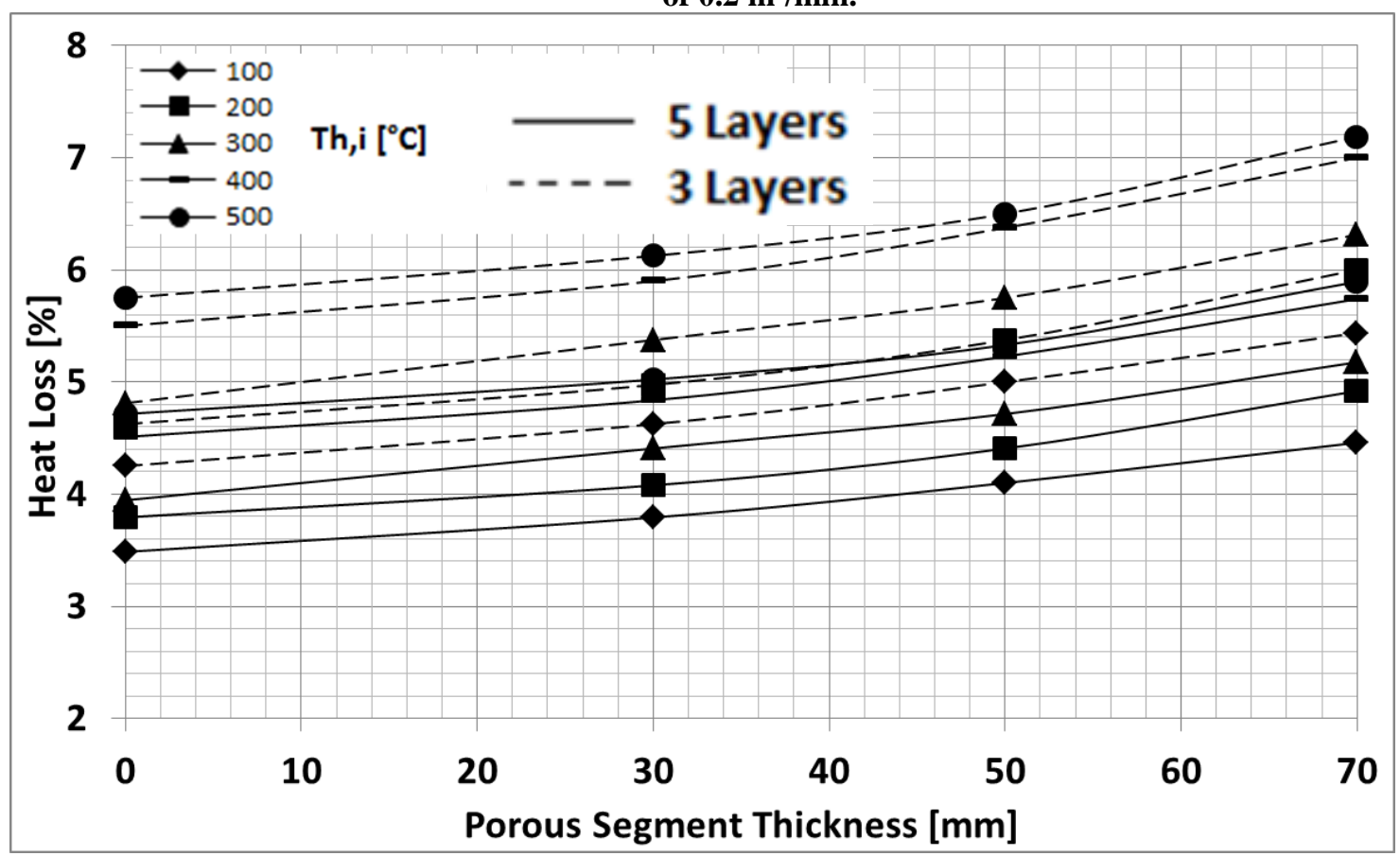

Fig. (18) Effects of $T_{h, i}$ and porous media thickness on Heat Loss at volume flow rate of $0.35 \mathrm{~m} 3 / \mathrm{min}$. 


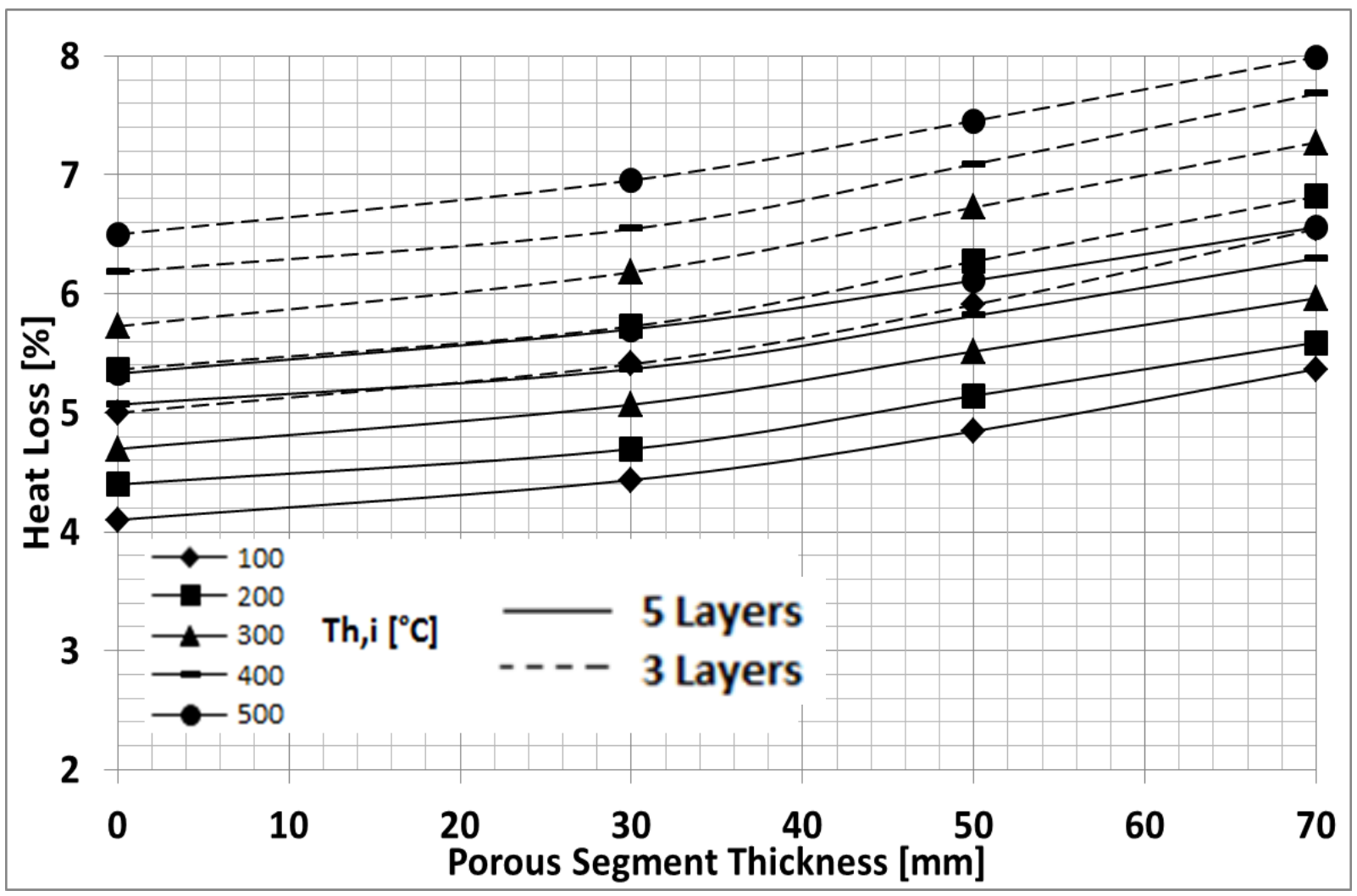

Fig. (19) Effects of $T_{h, i}$ and porous media thickness on Heat Loss at volume flow rate of $0.5 \mathrm{~m} 3 / \mathrm{min}$.

For the three layered heat exchanger, these percentages at the air volume flow rate of $0.2 \mathrm{~m}^{3 /} \mathrm{min}$ and $70 \mathrm{~mm}$ is 6.5 percent at hot air inlet temperatures of $500{ }^{\circ} \mathrm{C}$ and it was 8 when the air flow rate was raised to $0.5 \mathrm{~m} 3 / \mathrm{min}$. The corresponding increase in the heat loss is 23.1 percent.

\subsection{The Pressure Drop}

Since the cold air flows through a longer path that contains a higher number of segments of porous media and the connecting piping, it would face the highest pressure drop in the heat exchanger. Therefore the pressure drop across the cold- for the space considerations - air flow measured from the entry to the first heat recovery chamber till the exit of the heat exchanger from the bottom (last heat recovery one) is presented only in this paper. The results of the measurement of the pressure drop are presented in Figure 20 at different porous segment thicknesses, and different air volume flow rates for the tested five layered heat exchanger.

From Figure 20, it is noted that the pressure drop in the absence of porous segments represented by the points on the ordinate for zero thickness is negligible at the different air volume flow rates. In the presence of the porous inserts, the pressure drop that took place became measurable due to the higher friction between the air and the porous material as the air sweeps in the voids in addition to the pressure losses due to the sudden contractions and expansions at the entry and exit from the porous segments. The figure shows that at any flow rate, the pressure drop increases at a higher rate with the increase of the thickness of the inserted porous element due to the increase of the air path in the thicker segments. Furthermore, for the same porous insert size, the rate of the pressure drop rise becomes higher with the increase of the volume flow rate of the air stream due to the increased action of the above described pressure loss mechanisms with the flow rate.

The pressure losses encountered in the case of the three layered heat exchanger under the same operating conditions are also plotted in the same figure. 
For the five layered heat exchanger, the pressure drop reached $753.5 \mathrm{~Pa}$ at $30 \mathrm{~mm}$ thickness of porous material for $0.2 \mathrm{~m}^{3} / \mathrm{min}$. flow rate while it was $404 \mathrm{~Pa}$ for the three layered one at the same conditions. The pressure drop at the same flow rate $0.2 \mathrm{~m} / \mathrm{min}$. continued to increase by increasing the thickness of the porous segment till it reached $2192 \mathrm{~Pa}$ at $70 \mathrm{~mm}$ thickness for the present case and it was 1699 $\mathrm{Pa}$ for the three layered heat exchanger. The pressure drop increases from $1370 \mathrm{~Pa}$ at $30 \mathrm{~mm}$ porous material thickness to $3767.5 \mathrm{~Pa}$ at $70 \mathrm{~mm}$ porous material thickness at the same volume flow rate of 0.5 $\mathrm{m}^{3} / \mathrm{min}$. for the present heat exchanger, while it was 2545 for three layered one.

The pressure drop of the hot stream at these last conditions in the present case was $2410 \mathrm{~Pa}$ which represents about $64 \%$ of that of the cold stream.

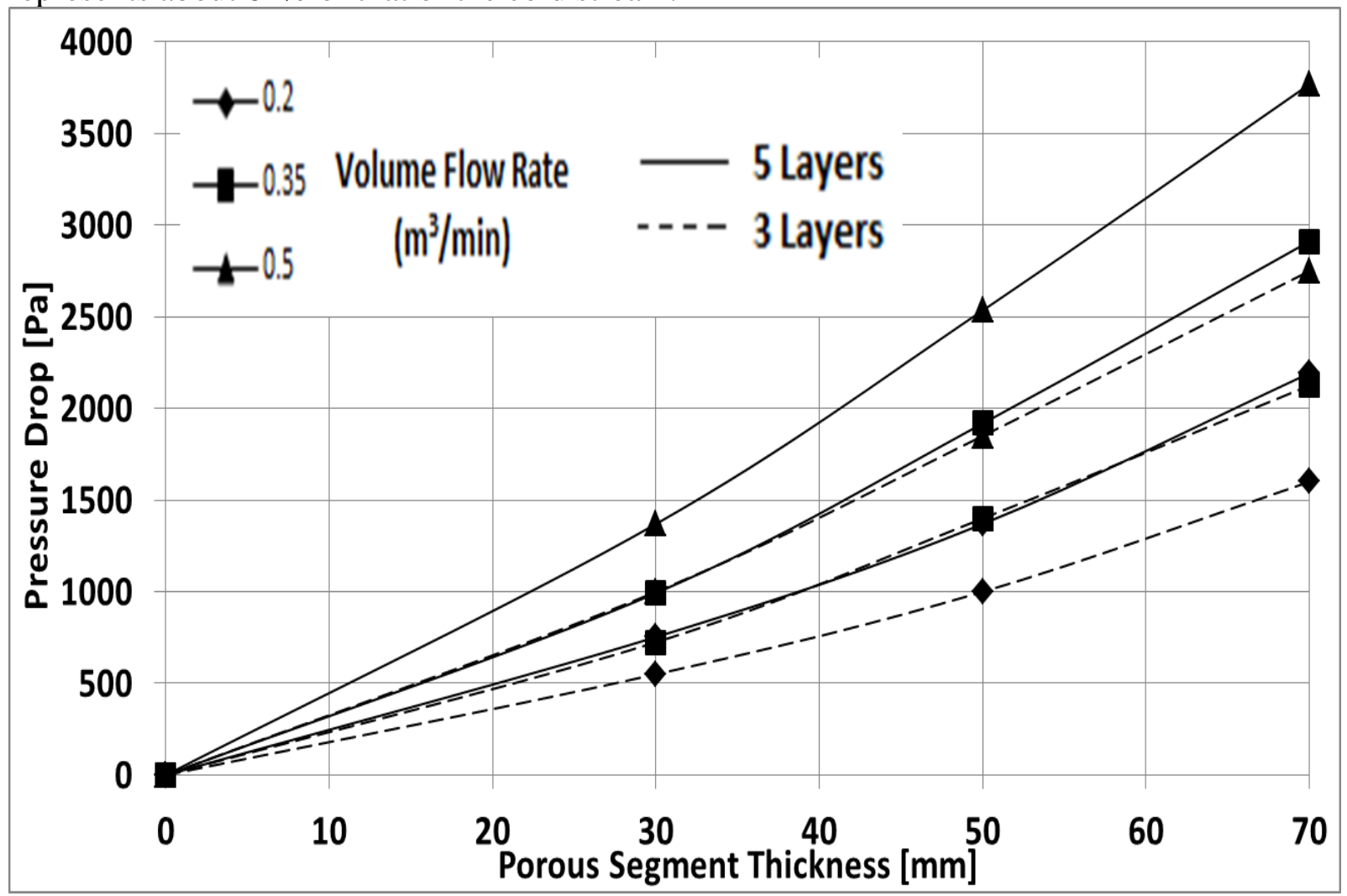

Fig. (20) Effects of porous media thickness on the pressure drop across the cold air chambers at different air volume flow rates

\section{THE HEAT RECOVERY RATE VERSUS THE POWER TO DRIVE THE FLOW}

In order to access the energy consumption to drive the air streams into the heat exchanger and the heat recovery from a hot exhaust gas stream to the colder one the case of the high hot air temperature $500^{\circ} \mathrm{C}$, the highest flow rate $0.5 \mathrm{~m}^{3} / \mathrm{hr}$ with the $70 \mathrm{~mm}$ porous inserted is considered. In this case the pressure drops for the present heat exchanger is 3767.5 pa and the rate of heat recovery is $936.33 \mathrm{~W}$. Assuming the overall efficiency of the blower drive the blower driving motor 50\%, the power consumption will be $0.53767 .5 /(0.5 * 60)=62.8$ Watt. The heat recovery rate is about 15 times that of the blower power.

The recorded energy is utilizing in heating processes in many applications, in process steam generations in heat recovery boilers and other processes. The use of the fossil fuels instead of the recovered energy is definitely more costly. Furthermore, the energy recovery helps to conserve the fossil energy sources and decreases its rate of depletion methods to improve its effectiveness than that obtained such as increasing the heat exchange surfaces within the chambers by installation of fins are being investigated. 


\section{EXPERIMENTAL INVESTIGATION OF THE PERFORMANCE OF FIVE LAYERED GAS TO GAS}

HEAT EXCHANGER WITH A POROUS MEDIUM

\section{Conclusion}

A series of experiments have been performed to investigate the thermo-hydrodynamic performance of a five layered gas to gas heat exchanger in laminar flow using porous media. The major findings of the present study are summarized as follows:

1- The use of porous material improves effectively the performance of this type of multi-layers heat exchangers. The improvement increases at any flow conditions with the increase of its porous segments' thickness. The degree of increase of the performance slows down and becomes asymptotic beyond a porous thickness $50 \mathrm{~mm}$.

2- This mode of increase of the performance applies to the increase in the cold air outlet temperature, the rate of heat recovery and the heat recovery ratio.

3-Under any flow rate and porous material thickness the increase in the energy recovery rate and the cold air outlet temper becomes higher as the hot air temperature attains larger values because the mechanism of converting the enthalpy of the hot gas into the internal energy of the porous material and the chambers enveloping walls, that is ultimately transferred to the cold air through the separating surfaces becomes more effectives. This situation prevails at all flow velocities and all porous medium thickness.

4- The step up of the flow velocity results in an augmentations of the convection heat transfer coefficient between the air and both of the porous medium and the interior surfaces of the cold and hot air chambers, which cause an increased rate of heat exchange to the enclosing walls of all chambers as well as the porous material .This effect accelerates the energy regeneration from the hot gas to the colder gas.

5- The gained enhancement of the above mentioned three performance criteria takes place on the expense a higher pressure drop which increase the power required to drive the two air streams through the heat exchanger, but the value of heat recovered from the exhaust gases in the present case is much more than the electric energy to drive the blower.

6- The above conclusions extend to the previously investigated performance of the three layered heat exchanger, but the performance of the five layered heat exchanger is superior to that of the three layered one. Estimates based on the announced economic cost of one $\mathrm{kWH}$ and the price of one million btu of natural gas energy showed that the heat recovered is less expensive

7- At the condition of highest performance $T_{h, i}=500^{\circ} \mathrm{C}$, volume flow rate $0.5 \mathrm{~m}^{3} / \mathrm{min}$ and $70 \mathrm{~mm}$ porous inserts which is the proper state for evaluation of results, the heat recovery rate for the five layered heat exchange was 186.73 Watt higher than that of the three layers while the pressure drop increased by 1222 pa which correspond to an increase is the motor electric consumption by about 20.32 Watt. The rate of increase of the recovered energy in the view of the increased blower power, due to the additional of one chamber in the path of each of the cold and hot air stream, justifies the use of the five layered one. Furthermore examination of the results showed that the heat recovery rate obtained by the three layer heat exchanger using $70 \mathrm{~mm}$ porous insert at volume flow rate $0.5 \mathrm{~m}^{3} / \mathrm{min} 749.41 \mathrm{Watt}$ can be obtained from the five layered one using the $30 \mathrm{~mm}$ porous segment and the same flow rate and hot air inlet temperature with a considerable reduction in the pressure drop equals 1175 pa and lower blower power.

8 - The percentage heat loss from each of the present heat exchanger and the three layer one increase with increasing the air inlet temperature, the volume flow rate and the porous material thickness, but at any similar operating condition the percentage heat loss from the present one is less than the corresponding percentage heat loss from the three layered one, being 6.56 and 8.035 respectively under the same previous operating conditions of $0.5 \mathrm{~m}^{3} / \mathrm{min}, 500^{\circ}$ Cand $70 \mathrm{~mm}$ porous material. 


\section{Nomenclature}

\begin{tabular}{|c|c|c|}
\hline$\dot{m}$ & 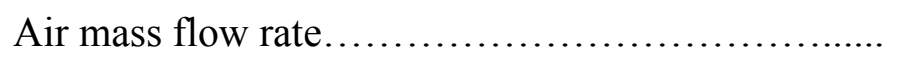 & $\mathrm{kg} / \mathrm{sec}$. \\
\hline$c_{p, c}$ & Cold air specific heat capacity at constant pressure... & $\mathrm{J} / \mathrm{kg} \cdot \mathrm{K}$ \\
\hline$c_{p, h}$ & Hot air specific heat capacity at constant pressure... & $\mathrm{J} / \mathrm{kg} . \mathrm{K}$ \\
\hline$T_{c, i}$ & Cold air inlet temperature..... & ${ }^{\circ} \mathrm{C}$ \\
\hline$T_{c, 0}$ & Cold air outlet temperature..... & ${ }^{\circ} \mathrm{C}$ \\
\hline $\bar{T}_{c}$ & Average cold air temperature.......................... & ${ }^{\circ} \mathrm{C}$ \\
\hline$T_{h, i}$ & Hot air inlet temperature............................. & ${ }^{\circ} \mathrm{C}$ \\
\hline$T_{h, o}$ & Hot air outlet temperature.......................... & ${ }^{\circ} \mathrm{C}$ \\
\hline $\bar{T}_{h}$ & Average hot air temperature......................... & ${ }^{\circ} \mathrm{C}$ \\
\hline$H_{R}$ & Heat recovery ratio.... & -- \\
\hline$Q_{h}$ & Rejected heat rate by the hot air...................... & Watt \\
\hline$Q_{c}$ & Heat recovery rate. Heat gained by the cold air....... & Watt \\
\hline$\% Q_{\text {loss }}$ & Percentage of heat loss......... & $\%$ \\
\hline HR1 & First Heat Recovery Section........................ & \\
\hline HR2 & $\begin{array}{l}\text { Second heat recovery section } \ldots \ldots \ldots \ldots \ldots \ldots \ldots \ldots \ldots \ldots \ldots \ldots \ldots \ldots \ldots \ldots \ldots \ldots \ldots \ldots \\
\text { Third heat recovery section } \ldots \ldots \ldots \ldots \ldots \ldots \ldots \ldots \ldots \ldots\end{array}$ & \\
\hline HR3 & & \\
\hline HT1 & First high temperature section..... & \\
\hline HT2 & Second high temperature section.. & \\
\hline $\mathrm{HL}$ & Low temperature chamber..... & \\
\hline
\end{tabular}

\section{REFERENCES}

[1] El-Hosseiny A.A., Experimental Study Of Three Layered Gas To Gas Heat Exchanger Using Porous Media, M.sc Thesis, Mechanical Power Department, Faculty of Engineering, Ain Shams University Cairo, 2012.

[2] Mehmet SOzen, Enhanced heat transfer in round tubes with porous inserts, International Journal of Heat and Fluid Flow, Vol. 17, No. 2, April 1996.

[3] A.A. Mohamad, Heat transfer enhancements in heat exchangers fitted with porous media, Part I: constant wall temperature,International Journal of Thermal Sciences, Volume 42, Issue 4, Pages 385-395, April 2003.

[4] Horng-Wen $\mathrm{Wu}$, Convective heat transfer over a heated square porous cylinder in a channel, International Journal of Heat and Mass Transfer, Volume 53, Issues 9-10, Pages 1927-1937, April 2010.

[5] Arunn Narasimhan, Laminar forced convection in a heat generating bi-disperse porous medium channel International Journal of Heat and Mass Transfer, Volume 54, Issues 1-3, Pages 636-644, 15 January 2011.

[6] H. Shokouhmand, The effect of porous insert position on the enhanced heat transfer in partially filled channels, International Communications in Heat and Mass Transfer, 10/2011,2011.

[7] Y. Tiana, C.Y. Zhao, A numerical investigation of heat transfer in phase change materials (PCMs) embedded in porous metals, International Journal of Heat and Mass Transfer, Volume 36, Issue 9, Pages 5539-5546, September 2011. 


\section{EXPERIMENTAL INVESTIGATION OF THE PERFORMANCE OF FIVE LAYERED GAS TO GAS}

HEAT EXCHANGER WITH A POROUS MEDIUM

[8] Shiang-WuuPerng, Horng-Wen Wu, Tswen-ChyuanJued, Numerical investigation of heat transfer enhancement on a porous vortex-generator applied to a block-heated channel, International Journal of Heat and Mass Transfer,Volume 55, Issues 11-12, Pages 3121-3137,May 2012.

[9] Saman Rashidi, Ali Tamayol, Mohammad Sadegh Valipour, NimaShokri, Fluid flow and forced convection heat transfer around a solid cylinder wrapped with a porous ring, International Journal of Heat and Mass Transfer,Volume 63, Pages 91-100, August 2013.

[10] A. Abou el-Mehrizi, M. Farhadi, K. Sedighi, M. Aghajani Delavar, Effect of fin position and porosity on heat transfer improvement in a plate porous media heat exchanger, Journal of the Taiwan Institute of Chemical Engineers, Volume 44, Issue 3, Pages 420-431, May 2013.

[11] Yasser Mahmoudi, Nader Karimib, Numerical investigation of heat transfer enhancement in a pipe partially filled with a porous material under local thermal non-equilibrium condition, International Journal of Heat and Mass Transfer, Volume 68, Pages 161-173, January 2014.

[12] Mahboobe Mahdavi et al, Entropy generation and heat transfer numerical analysis in pipes partially filled with porous medium, International Journal of Heat and Mass Transfer, Volume 79, Pages 496-506, December 2014.

[13] Fumei Rong, Numerical study of heat transfer enhancement in a pipe filled with porous media by axisymmetric TLB model based on GPU, International Journal of Heat and Mass Transfer, Volume 70, Pages 1040-1049, March 2014.

[14] Pei-Xue Jiang, Thermal hydraulic performance of small scale micro-channel and porous-media heatexchangers, International Journal of Heat and Mass Transfer, Volume 44, Issue 5, Pages 1039-1051,March 2001.

[15] Pei-Xue Jiang, Experimental research on convection heat transfer in sintered porous plate channels, International Journal of Heat and Mass Transfer, Volume 47, Issues 10-11, Pages 2085-2096, May 2004.

[16] Kang-HoonKo, N.K. Anand, Use of porous baffles to enhance heat transfer in a rectangular channel, International Journal of Heat and Mass Transfer, Volume 46, Issue 22, Pages 4191-4199, October 2003.

[17] N. Delalic, Porous media compact heat exchanger unit-experiment and analysis, Experimental Thermal and Fluid Science, Volume 28, Issues 2-3, Pages 185-192, January 2004.

[18] C. Hutter, Heat transfer in metal foams and designed porous media, Chemical Engineering Science, Volume 66, Issue 17, Pages 3806-3814, 1 September 2011.

[19] T. Tomimura, Experimental study on multi-layered type of gas to gas heat exchanger using porous media, International Journal of Heat and Mass Transfer, Volume 47, Issue 21, Pages 4615-4623, October 2004.

[20] Bogdan I. Pavel, An experimental and numerical study on heat transfer enhancement for gas heat exchangers fitted with porous media, International Journal of Heat and Mass Transfer, Volume 47, Issue 23, Pages 4939-4952, November 2004.

[21] G. Hetsroni, Sintered porous medium heat sink for cooling of high-power mini-devices, International Journal of Heat and Fluid Flow, Volume 27, Issue 2, Pages 259-266, April 2006.

[22] S. A. GandjalikhanNassab, Thermal Behavior of A New Type of Multi-Layered Porous Air Heater, Journal of Heat Transfer Vol. 19, No. 1, November 2006.

[23] Z.F. Huang, Enhancing heat transfer in the core flow by using porous medium insert in a tube, International Journal of Heat and Mass Transfer, Volume 53, Issues 5-6, Pages 1164-1174, February 2010.

[24] G. Venugopal, Experimental study of mixed convection heat transfer in a vertical duct filled with metallic porous structures, International Journal of Thermal Sciences, Volume 49, Issue 2, Pages 340-348, February 2010

[25] Chainarong Chaktranond, Analysis of heat and mass transfer enhancement in porous material subjected to electric fields (effects of particle sizes and layered arrangement), Experimental Thermal and Fluid Science, Volume 34, Issue 8, Pages 1049-1056, November 2010.

[26] Khaled Al-Salem, Hakan F. Oztop, Suhil Kiwanc, Effects of porosity and thickness of porous sheets on heat transfer enhancement in a cross flow over heated cylinder, International Communications in Heat and Mass Transfer, Volume 38, Issue 9, Pages 1279-1282,November 2011. 